\title{
A review of the polysaccharide, protein and selected nutrient content of Auricularia, and their potential pharmacological value
}

\author{
Bandara $\mathrm{AR}^{1,2}$, Rapior $\mathrm{S}^{3}$, Mortimer $\mathrm{PE}^{4}$, Kakumyan $\mathrm{P}^{1,2}$, Hyde $\mathrm{KD}^{1,2,4,5}, \mathrm{Xu} \mathrm{J}^{4,5}$ \\ ${ }^{1}$ Center of Excellence in Fungal Research, Mae Fah Luang University, Chiang Rai 57100, Thailand \\ ${ }^{2}$ School of Science, Mae Fah Luang University, Chiang Rai 57100, Thailand \\ ${ }^{3}$ Laboratoire de Botanique, Phytochimie et Mycologie, Faculté de Pharmacie, CEFE CNRS - Université de \\ Montpellier - Université Paul-Valéry Montpellier - EPHE - IRD, B.P. 14491, 15 avenue Charles Flahault, 34093 \\ Montpellier Cedex 5, France \\ ${ }^{4}$ Key Laboratory for Plant Diversity and Biogeography of East Asia, Kunming Institute of Botany, Chinese Academy of \\ Science, Kunming 650201, Yunnan, China \\ ${ }^{5}$ World Agroforestry Centre, East and Central Asia, Kunming 650201, Yunnan, China
}

Bandara AR, Rapior S, Mortimer PE, Kakumyan P, Hyde KD, Xu J 2019 - A review of the polysaccharide, protein and selected nutrient content of Auricularia, and their potential pharmacological value. Mycosphere 10(1), 579-607, Doi 10.5943/mycosphere/10/1/10

\begin{abstract}
Auricularia is a key genus among edible macrofungi, sourced as either wild or cultivated mushrooms. Auricularia species are utilized as nutrient-rich foods and medicinal resources, with particular prominence in Traditional Asian Medicine. Cultivated Auricularia species can be grown under a wide range of conditions, allowing for production volumes worldwide. Although Auricularia species are used predominantly within the food industry, there is strong potential for their use in the production of therapeutic drugs, thus making it necessary to identify relevant bioactive compounds and further our understanding of its pharmacological properties. Carbohydrates are the major nutritional constituent of edible Auricularia species in addition to proteins, fat, fiber, ashes vitamins and minerals. This review discusses polysaccharides as one of the major active compounds found in edible Auricularia species in relation to their nutritional value, extraction methods, and pharmacological properties. Current methods of evaluating the pharmacological effects of compounds derived from Auricularia include in vitro assays, in vivo animal models, as well as several human clinical trials. Potential medical applications for these compounds include the production of novel therapeutic drugs for treating diseases such as cancer, diabetes, and cardiovascular disorders.
\end{abstract}

Key words - Cancer - Cardiovascular disorders - Constipation - Diabetes - Medicinal mushrooms - Metabolic Syndrome - Polysaccharides

\section{Introduction}

Fungi have long been regarded as a source of natural medicine, and mushrooms used for medicinal purposes feature prominently in Traditional Asian Medicine (Hapuarachchi et al. 2018b, Hyde et al. 2019). Over the last four decades, various therapeutic activities have been reported as a result of extensive research on mushrooms using in vitro assays, in vivo animal models, and, in some cases, human clinical studies; these include but are not limited to: anti-cancer, anti-diabetic, anti-malarial, anti-microbial, antioxidant, anti-tumor, anti-viral, neuroprotective, and 
hypocholesterolemic properties (De Silva et al. 2012a, b, 2013, Dalonso et al. 2015, Thongbai et al. 2015, Hapuarachchi et al. 2016, Klupp et al. 2016, Wasser 2017, Hapuarachchi et al. 2018a). In the past mushrooms were primarily used as a food source (Dupont et al. 2017). The aforementioned published reports have changed our perception of mushrooms as a functional food, with mushrooms increasingly viewed as a medicinal resource (Thawthong et al. 2014, Hapuarachchi et al. 2018a). With the use of mushrooms both as a food and medicinal source, global mushroom production has increased more than 30-fold since 1978 (Royse et al. 2017). The increment in global mushroom porudction also coincides with a simultaneous increase (1.7-fold) in the global human population (Royse et al. 2017), presumably due to the importance of mushrooms as a food source (Chang \& Wasser 2017). There are two major groups of edible fungi; a) Ascomycetes, which include Discomycetes (Cup fungi) and the famous Tuberales or truffles, and b) Basidiomycetes, which include Phragmobasidiomycetidae encompassing three orders: Auriculariales, Septobasidiales and Tremellales (Alexander 2013). Among these mushrooms, the order Auriculariales (previously known as "Jelly fungi") contain a number of species that have value as both edible and medicinal mushrooms (Alexander 2013).

Auricularia Bull. is an important genus which accounts for approximately $17 \%$ of world mushroom production and is the third most cultivated mushroom genus after Lentinula (22\%) and Pleurotus (19\%) (Royse et al. 2017). Besides its significance as a major food source, Auricularia species can be exploited as medicinal resources for the development of novel therapeutic drugs for many human diseases, including potential for cancer treatment ( $\mathrm{Wu}$ et al. 2014). The bioactive compounds of Auricularia species which primarily include polysaccharides, are known for their anti-tumor, antioxidant, anti-coagulant and immunomodulating properties ( $\mathrm{Ma}$ et al. 2018). Knowledge of the bioactive compounds of Auricularia is crucial for furthering our understanding of their pharmacology and could lead to the development of novel therapeutic drugs. This review provides a synopsis of the biochemistry and pharmacology of Auricularia species.

\section{Cultivation of Auricularia}

Auricularia auricula-judae (known as black fungus or wood ear mushroom) has a history of $\sim 2100$ years of cultivation in China (Yao et al. 2018). Currently, A. cornea and A. heimuer (Fig. 1) are commercially cultivated in China, Indonesia, Malaysia, Philippines, Thailand and Vietnam (Chang \& Lee 2004, Duc 2005, Tapingkae 2005, Peng 2008, Reyes et al. 2009, Irawati et al. 2012, Razak et al. 2013, Wu et al. 2014). China is the main producer of cultivated Auricularia, and approximately 6.3 billion $\mathrm{kg}$ were produced in 2017, which made it the second most widely cultivated mushroom in that country (Chinamushroombusinessnetwork 2018).

Several culture media with different nutritional profiles, as well as optimal temperatures and $\mathrm{pH}$ ranges, are currently used to cultivate Auricularia, i.e., Czapek-dox, Glucose Peptone, Malt Extract Agar (MEA), Mesangial Cell Medium (MCM), Potato Dextrose Agar (PDA), Yeast Extract Agar (YEA), Yeast Mannitol Agar (YMA), and Leonian medium (Yu et al. 2013, Jo et al. 2014). The biomass production of edible Auricularia requires optimal growth conditions for their mycelia. For instance, A. auricula-judae was reported to be grown optimally on PDA and MCM at $25-30^{\circ} \mathrm{C}$ and pH 6-9, but not on Czapek dox or Leonian (Jo et al. 2014). Auricularia villosula mycelium can be cultivated on a mix of potato juice, sucrose, soybean powder and $0.5 \% \mathrm{PO}_{4}^{-3}$ under optimal temperature $\left(30^{\circ} \mathrm{C}\right)$ and $\mathrm{pH} 8$ conditions to produce fruiting bodies that are very similar to those found in nature (Zhang et al. 2018b). Spawn production, generated from the tissue culturing of mycelia, followed by fruiting body production (either on logs, whether wood or artificial, or through the poly bag method), are the main cultivation methods practiced (Priya et al. 2016). Lowcost production of Auricularia species is carried out in many Southeast Asian countries by using compost and agro-waste products. For example, compost consisting primarily of corncobs, rice straw, broadleaf tree sawdust, and cottonseed bran with plaster stone, wheat bran, rice bran, and quick lime as supplementary materials, was successfully used to cultivate A. auricula-judae in India (Verma \& Verma 2017). Similarly, in Taiwan, low-cost agro-waste products, i.e., cotton 
waste, rice straw, wood chips, and sawdust are commonly used for the production of Auricularia species (Peng 2008).

Optimal culture conditions (temperature, $\mathrm{pH}$ ) with nutritionally rich supplementation are necessary for increasing the yield as well as commercial quality of edible mushrooms (Carrasco et al. 2018). The substrate supplementation is known to impact the nutritional content of Auricularia. For example, the use of $60 \%$ sugarcane bagasse resulted in the highest nutrient output (carbohydrates, protein, ash, and fat) from A. polytricha in contrast to other agro-waste substrates such as rice straw, and rice husk (Wu et al. 2017). On the other hand, $A$. auricula grown on maize cobs and wheat bran showed higher nutrient content (cellulose, proteins and moisture) in contrast to the counterparts grown on saw dust and rice bran in Kenya (Onyango et al. 2011). The dry biomass weight that represent the content of moisture, crude proteins, ash and carbohydrates has also been reported to be dependent on the type of nutrient medium used for their cultivation. For instance, nitrogen source that was most favorable for A. polytricha growth and highest dry biomass production was yeast extract in contrast to reducing impact from tryptone, beef extract and peptone (Hassan \& Medany 2012).

The wide range of growing conditions and methods used for production also provides implications on the environmental plasticity of Auricularia sp. It also coincides with the high degree of interspecies and intraspecies genetic diversity. Past studies have shown that intraspecies and interspecies genetic diversity is quite high, and this may impact their breeding and cultivation conditions (Yan et al. 2004, Li et al. 2007, Du et al. 2013, Li et al. 2014). In order to address this intraspecies genetic variability, new techniques employing random amplified polymorphic DNA (RAPD) fingerprinting analysis have been shown to be highly successful in determining the genetic diversity of $A$. auricula and A. polytricha strains, and have been suggested as an effective method for determining breeding techniques (Yan et al. 2004).
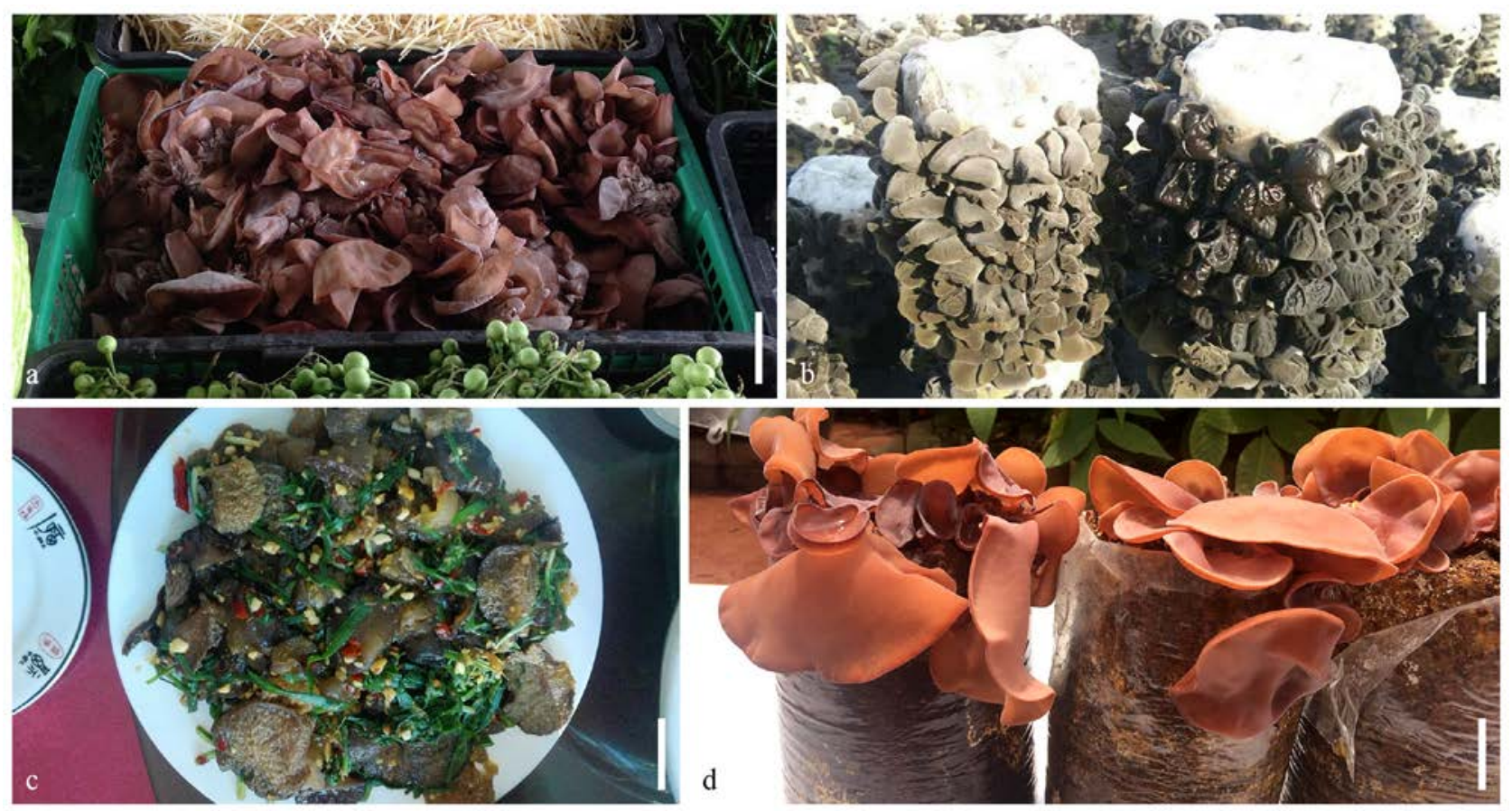

Figure 1 - Edible species of Auricularia in Asia. a Auricularia cornea (Phetchaburi, Thailand). b Auricularia heimuer (Mudanjiang, Heilonjiang, China). c Auricularia delicata (Honghe, Yunnan, China). d Auricularia thailandica (Kunming, Yunnan, China). Scale bars: a-d $=3 \mathrm{~cm}$. These pictures are copyright of Asanka R. Bandara.

\section{Auricularia as an edible mushroom}

Auricularia is a cosmopolitan genus, comprised of eighteen species (Bandara et al. 2017b) of which Auricularia auricula-judae (Bull.: Fr.) Quél., [= A. auricula (L. ex Hook.) Underw.], A. 
cornea (Ehrenb.) Fr., A. delicata (Mont. ex Fr.) Henn., A. fuscosuccinea (Mont.) Henn., A. heimuer F. Wu, B.K. Cui, Y.C. Dai, A. thailandica Bandara \& K.D. Hyde and A. villosula Malysheva have been reported as edible species (Fig. 1) (Zent et al. 2004, Wu et al. 2014, Sekara et al. 2015, Zhang et al. 2015a, Bandara et al. 2017a, b, Kamalebo et al. 2018, Zhang et al. 2018b). The characteristic flavor, along with the slippery and crunchy texture of the gelatinous fruiting body of Auricularia species accounts for the popularity and high consumption in China and Southeast Asia (Misaki \& Kakuta 1995, Cheung 2013, Jo et al. 2014).

\section{Nutrient composition of Auricularia}

The nutritional content of edible Auricularia varies according the species. On average, dried Auricularia have an approximate composition of $79.9-93.2 \%$ carbohydrates, $6.5-13 \%$ crude proteins, 9.9-17.9\% total soluble sugars, $0.48-4.5 \%$ crude fat (lipid), and 3.5-12.5\% crude fiber (Table 1) (Crisan \& Sands 1978, Cheung 1997, Mau et al. 1998, Chen et al. 2011, Kadnikova et al. 2015, Bandara et al. 2017a, USDA 2018a). Carbohydrates are the major components in Auricularia. For example, proximate composition of A. auricula-judae represents carbohydrate: 81.0\%, crude protein: $8.1 \%$, crude fat: $1.5 \%$, crude fiber: $6.9 \%$, and ash: $9.4 \%$ (Chang \& Hayes 2013, Cheung 2013).

Digestible nutrients found in edible mushrooms vary depending on the species, age, and their cultivation methods, and include major nutrients such as carbohydrates, proteins, and minerals (Ca, $\mathrm{Na}, \mathrm{K}$, and Mg) (Kadnikova et al. 2015). The digestible carbohydrates are primarily composed of polysaccharides and include water soluble mannans and glucans, pectins, chitin, and cellulose whose digestibility may vary depending on the percentage of these contents (Kadnikova et al. 2015). The extent of lignification also determine the digestibility. However, the non-digestible nutrients such as non-starch polysaccharides represented by fibers are useful as a food source with higher health benefits (Cheung 2013). In addition to these macronutrients, edible mushrooms provide a good source of micronutrients for which most human diets are deficient such as $\mathrm{Cu}$, Se and Zn (Carrasco et al. 2018). Moreover, Auricularia species are known to be a source of many minerals that may help to solve human nutrient deficiencies including $\mathrm{Ca}, \mathrm{Cu}, \mathrm{Cr}, \mathrm{Fe}, \mathrm{Mg}, \mathrm{Mn}, \mathrm{Ni}$ and Zn (Shin et al. 2007, Kadnikova et al. 2015). As with other wild and cultivated edible mushrooms (Kalač 2010, 2013, Wang et al. 2014), potassium is the most prevalent macro-element within Auricularia species (Afiukwa et al. 2013, Bandara et al. 2017a, USDA 2018a, b), which are typically nutrient-rich with other macro-elements including $\mathrm{Ca}, \mathrm{Mg}$, Na and P (Table 3) (Afiukwa et al. 2013, Bandara et al. 2017a). Among micro-elements, high concentrations of Fe and Zn have been reported in Auricularia (50-200 mg/kg), while those of $\mathrm{Co}, \mathrm{Cr}, \mathrm{Cu}, \mathrm{Mn}$ and $\mathrm{Ni}$ were found to be less than $20 \mathrm{mg} / \mathrm{kg}$ of dry weight (Kadnikova et al. 2015, Bandara et al. 2017a). Studies also suggest an intraspecies variability of micronutrients content of $\mathrm{Mg}, \mathrm{Mn}, \mathrm{Zn}, \mathrm{Ni}, \mathrm{Cr}, \mathrm{Sr}$ in $\mathrm{A}$. auricula where as the Fe levels were quite similar among all the strains with a maximum concentration of $285 \mu \mathrm{g} / \mathrm{g}$ ( $\mathrm{Li}$ et al. 2018).

Auricularia is also a rich source of dietary fiber (Cheung 2013). Most of the carbohydrates in Auricularia have been reported to be indigestible polysaccharides, such as $\beta$-glucans and mannans (Misaki \& Kakuta 1995, Mironczuk-Chodakowska et al. 2017). Auricularia species have been shown to contain a higher fiber content than other commercially available mushrooms such as Agaricus bisporus and Ganoderma lucidum (Misaki \& Kakuta 1995, Cheung 1997).

Auricularia species have very low soluble sugar content, which is reflected in their flavor (Mau et al. 1998). Furthermore, unlike other edible mushrooms, Auricularia have a low fat content (Cheung 2013), and 60\% of the fatty acids of harvested wild Auricularia are unsaturated (Kavishree et al. 2008). Low-fat diets with a high content of unsaturated fatty acids are recommended for people with high blood cholesterol, making Auricularia an ideal food choice for them (Lichtenstein et al. 2006, Chen et al. 2011).

Quantities of crude proteins in Auricularia species are lower than in most wild and cultivated edible mushroom species (Fan et al. 2006, Liu et al. 2009, Cheung 2013, Jia et al. 2017). Common edible mushrooms in the market, such as A. bisporus, Lentinula edodes, and Pleurotus spp. contain 
10.5-34.8\% of crude protein, whereas A. auricula-judae contains $8.1 \%$ of crude protein (Chang \& Miles 2004, Cheung 2013). Edible species of Auricularia provide 17-18 amino acids, including eight essential amino acids and three semi-essential amino acids (arginine, cysteine, and tyrosine), all of which are of value for pediatric nutrition (Table 2) (Afiukwa et al. 2015, Bandara et al. 2017a, Ohiri \& Bassey 2017). Glutamic acid was the most abundant amino acid present in Auricularia, whereas sulfur-containing amino acid (cysteine, methionine) quantities were shown to be relatively low (Table 2) (Afiukwa et al. 2015, Bandara et al. 2017a, Ohiri \& Bassey 2017).

Table 1 Nutrient content including total carbohydrate, fat, fiber, protein, free amino acids and total soluble sugars of Auricularia auricula-judae, A. fuscosuccinea, A. polytricha and A. thailandica (\% of dry weight).

\begin{tabular}{|c|c|c|c|c|c|c|c|}
\hline $\begin{array}{l}\text { Auricularia } \\
\text { species }\end{array}$ & $\begin{array}{l}\text { Total } \\
\text { carbohydrate }\end{array}$ & Fat & Fiber & Protein & $\begin{array}{l}\text { Free } \\
\text { amino } \\
\text { acids }\end{array}$ & $\begin{array}{l}\text { Total } \\
\text { soluble } \\
\text { sugars }\end{array}$ & References \\
\hline $\begin{array}{l}\text { Auricularia } \\
\text { auricula-judae }\end{array}$ & 81.0 & $1.5,1.7$ & $3.5,6.9$ & $12.5,11.5$ & - & 10.2 & $\begin{array}{l}\text { (Crisan \& Sands } \\
\text { 1978, Aletor 1995, } \\
\text { Kadnikova et al. } \\
\text { 2015) }\end{array}$ \\
\hline $\begin{array}{l}\text { Auricularia } \\
\text { fuscosuccinea } \\
\text { (b) }\end{array}$ & 71.2, & 4.5 & 11.7 & 8.6 & 0.12 & 9.9 & (Mau et al. 1998) \\
\hline $\begin{array}{l}\text { Auricularia } \\
\text { fuscosuccinea } \\
\text { (w) }\end{array}$ & 68.9 & 4.5 & 12.5 & 12.5 & 0.07 & 10.9 & (Mau et al. 1998) \\
\hline $\begin{array}{l}\text { Auricularia } \\
\text { polytricha }\end{array}$ & $88.1,91.1$ & $\begin{array}{l}0.48 \\
0.5\end{array}$ & 3.6 & 6.5 & 0.05 & 17.9 & $\begin{array}{l}\text { (Mau et al. 1998, } \\
\text { USDA 2018a) }\end{array}$ \\
\hline $\begin{array}{l}\text { Auricularia } \\
\text { thailandica }\end{array}$ & - & 2.93 & 4.62 & 12.99 & 1.13 & 16.23 & $\begin{array}{l}\text { (Bandara et al. } \\
\text { 2017a) }\end{array}$ \\
\hline
\end{tabular}

b: brown strain; w: white strain; nitrogen to protein conversion factor: $\mathrm{N} \times 6.25$.

Table 2 Amino acid contents of Auricularia auricula-judae, A. fuscosuccinea, A. polytricha and A. thailandica (\% of dry weight).

\begin{tabular}{|c|c|c|c|c|c|}
\hline Amino acid & $\begin{array}{l}\text { Auricularia } \\
\text { auricula-judae }\end{array}$ & $\begin{array}{l}\text { Auricularia } \\
\text { fuscosuccinea (b) }\end{array}$ & $\begin{array}{l}\text { Auricularia } \\
\text { fuscosuccinea } \\
\text { (w) }\end{array}$ & $\begin{array}{l}\text { Auricularia } \\
\text { polytricha }\end{array}$ & $\begin{array}{l}\text { Auricularia } \\
\text { thailandica }\end{array}$ \\
\hline ALA alanine & 3.44 & 0.24 & 0.04 & 0.11 & 0.53 \\
\hline ARG arginine & 3.37 & 0.13 & 0.08 & 0.04 & 0.63 \\
\hline ASP aspartic acid & 3.00 & 0.12 & 0.06 & 0.02 & 0.35 \\
\hline CYS cysteine & 0.30 & - & - & - & 0.15 \\
\hline GLU glutamic acid & 5.53 & 0.14 & 0.16 & 0.03 & 0.92 \\
\hline GLY glycine & 1.32 & 0.02 & 0.02 & 0.01 & 0.29 \\
\hline HIS histidine $^{\mathrm{e}}$ & 0.96 & - & - & 0.03 & 0.52 \\
\hline ILE isoleucine $^{\mathrm{e}}$ & 1.03 & 0.04 & 0.03 & 0.04 & 0.40 \\
\hline LEU leucine ${ }^{\mathrm{e}}$ & 1.94 & - & - & 0.05 & 0.73 \\
\hline LYS lysine $\mathrm{e}^{\mathrm{e}}$ & 1.22 & 0.13 & nd & 0.13 & 0.58 \\
\hline MET methionine $^{\mathrm{e}}$ & 0.35 & - & - & 0.007 & 0.01 \\
\hline PHE phenylalanine ${ }^{\mathrm{e}}$ & 1.06 & 0.07 & 0.02 & 0.04 & 0.44 \\
\hline PRO proline & 0.20 & - & - & - & 0.36 \\
\hline THR threonine ${ }^{\mathrm{e}}$ & 1.74 & 0.16 & 0.16 & 0.03 & 0.58 \\
\hline TRP tryptophan & - & 0.02 & 0.01 & 0.01 & - \\
\hline TYR tyrosine & 1.03 & 0.03 & 0.06 & 0.02 & 0.21 \\
\hline SER serine & 2.07 & 0.08 & 0.16 & 0.02 & 0.31 \\
\hline VAL valine $^{\mathrm{e}}$ & 1.25 & 0.06 & 0.03 & 0.03 & 0.97 \\
\hline References & $\begin{array}{l}\text { (Afiukwa et al. } \\
\text { 2015) }\end{array}$ & (Mau et al. 1998) & $\begin{array}{l}\text { (Mau et al. } \\
\text { 1998) }\end{array}$ & $\begin{array}{l}\text { (Mau et al. 1998, } \\
\text { Razak 2013) }\end{array}$ & $\begin{array}{l}\text { (Bandara et al. } \\
\text { 2017a) }\end{array}$ \\
\hline
\end{tabular}

b: brown strain; w: white strain; nd: not detected; ${ }^{\text {e: }}$ essential amino acid. 
Table 3 Mineral contents of Auricularia auricula-judae, A. polytricha and A. thailandica (mg/kg).

\begin{tabular}{llll}
\hline Mineral element & $\begin{array}{l}\text { Auricularia auricula- } \\
\text { judae }(\mathbf{d w})\end{array}$ & $\begin{array}{l}\text { Auricularia polytricha } \\
\text { (fw) }\end{array}$ & $\begin{array}{l}\text { Auricularia thailandica } \\
\text { (dw) }\end{array}$ \\
\hline $\mathrm{Ca}$ & 16000 & 160 & 885 \\
\hline $\mathrm{K}$ & 12000 & 430 & 13780 \\
\hline $\mathrm{Mg}$ & 2000 & 250 & 895 \\
\hline $\mathrm{Na}$ & 8000 & 90 & 113 \\
\hline $\mathrm{P}$ & - & 140 & 3880 \\
\hline $\mathrm{Cr}$ & $\leq 20$ & - & 4 \\
\hline $\mathrm{Cu}$ & $\leq 20$ & 4 & 6 \\
\hline $\mathrm{Fe}$ & 200 & 5 & 64 \\
\hline $\mathrm{Mn}$ & $\leq 20$ & 1 & 8 \\
\hline $\mathrm{Ni}$ & $\leq 20$ & - & 7 \\
\hline $\mathrm{Zn}$ & 60 & 6 & 49 \\
\hline & (Kadnikova et al. 2015) & (USDA 2018a) & (Bandara et al. 2017a) \\
\hline
\end{tabular}

dw: dry weight; fw: fresh weight.

\section{Medicinal properties and pharmacological applications of Auricularia}

Auricularia species have long been used as traditional remedies in Asia, European folk cultures, African communities, as well as among indigenous groups such as the Māori (New Zealand) and Tzeltal (Mexico) (Fuller et al. 2004, Guzmán 2008, Pala et al. 2013, Sekara et al. 2015, Teke et al. 2018). As an example, A. cornea has been used as a traditional medicine to heal poisoning by toxic plants among Māori tribes (Fuller et al. 2004, Fuller et al. 2005), and certain Auricularia species have been used by the Tzeltal people to treat anxiety as part of their traditional medicine (Guzmán 2008). However, the application of Auricularia for treatment of human disease has moved beyond the domain of traditional medicines and is now being incorporated into Western medicine.

In A. auricula-judae, the major polysaccharide constituents of the fruiting body contained water-soluble glucan I with $\beta$-D-configuration (Fig. 2.1); alkali-insoluble glucan II with $\beta$-Dglucosidic linkages (Fig. 2.2); and acidic heteropolysaccharides, which were a mix of xylose, mannose, glucose and glucuronic acid (1.0: 4.1: 1.3: 1.3) (Table 4) (Sone et al. 1978). Watersoluble glucan I with branched $(1 \rightarrow 3)-\beta$-D-glucan has been shown to have anti-tumor effects against sarcoma in mice (Table 4) (Misaki et al. 1981).

Numerous studies have reported on the medicinal properties and pharmacological activities of isolated compounds or different solvent extracts from Auricularia (Sekara et al. 2015). In particular, the anti-coagulant (Yoon et al. 2003), anti-inflammatory (Ukai et al. 1983a, Kiho et al. 1985, Damte et al. 2011), anti-microbial (Gbolagade \& Fasidi 2005), antioxidant (Sun et al. 2010, Bandara et al. 2017a), anti-tumor (Misaki et al. 1981, Ukai et al. 1983b), anti-viral (Nguyen et al. 2012a), and immunomodulatory (Sheu et al. 2004) activities of Auricularia have been demonstrated. In addition, Auricularia has the potential to treat both hypoglycemia (Yuan et al. 1998a) and hypolipidemia (Reza et al. 2015), as well as lowering cholesterol levels (Table 5) (Misaki \& Kakuta 1995, Chen et al. 2008, Zhao et al. 2015). Furthermore, research has shown that Auricularia mushrooms have the ability to modulate interstitial microbiomes; for instance, $A$. auricula had a negative impact on Fusobacteriales and a positive impact on Bifidobacteriales and Bacteroidales, two of the most important probiotics (Zhao et al. 2018).

The high fiber content of Auricularia has been shown to contribute to preventing gastric disorders (Misaki \& Kakuta 1995), such as functional constipation in humans (Kim et al. 2004). Fibers from Auricularia have also been used to treat gastric disorders associated with nausea (Khan et al. 2016). In addition to helping with gastric disorders, the dietary fibers in Auricularia are known to be bioactive compounds with antioxidant and hypocholesterolemic properties (Cheung 1996).

Previous studies have mentioned the promising effects of Auricularia as an anti-cancer and anti-tumor agent (Table 5) (Misaki \& Kakuta 1995, Ma et al. 2010, Song \& Du 2012a). Due to the 
growing evidence that Auricularia species are able to treat gastric disorders, compounds derived or extracted from Auricularia have been used in anti-gastrointestinal cancer therapy, including treatments for colorectal, gastric, liver, pancreatic, and esophageal cancer (Yu et al. 2014, Ma et al. 2018). For example, $A$. polytricha crude polysaccharide extract with a $(1 \rightarrow 3)$-linked- $\beta$-Dglucopyranosyl and $(1 \rightarrow 3,6)$-linked- $\beta$-D-glucopyranosyl residue backbone $(2: 1$ ratio) and a terminal $(1 \rightarrow)-\beta$-D-glucopyranosyl was shown to inhibit S180 sarcoma cancer transplanted in mice, providing evidence of its anti-cancer properties (Song \& Du 2010). Similarly, sarcoma 180 solid tumors could be inhibited by water soluble and ethanol extracts of polysaccharides from $A$. auricula-judae through the inhibition of acinar cell carcinoma proliferation and tumor cell apoptosis mediated by reduced $\mathrm{Bcl}-2$ gene expression and increased Bax expression. A recent meta-analysis provided strong evidence for the use of polysaccharides from A. auricula (Huaier) in anti-gastrointestinal cancer therapy (Ma et al. 2018). Fermentation of $A$. auricula followed by hot water extraction gives rise to Huaier cream, which is then mixed with powdered sugar, dextrin, and auxiliary materials to generate Huaier granule. Ma et al. (2018) describes Huaier granules as possessing not only anti-cancer properties but also antioxidant and anti-coagulant activities (Ma et al. 2018). The article cites several studies that have used either the Huaier granule or Huaier cream to treat esophageal, pancreatic, gastric, colorectal and hepatocellular cancers (Ma et al. 2018). Moreover, A. auricula-judae ethanol extract has been used to generate a dichloromethane fraction, which contains diazene, a compound known to be used in treating tumors. The dichloromethane fraction was able to inhibit bronchoalveolar cancer and gastric cancer cells by controlling the levels of tumorigenic genes, including p53 overexpression and Bcl-2 downregulation, to induce cytotoxicity and apoptosis, respectively (Reza et al. 2014).

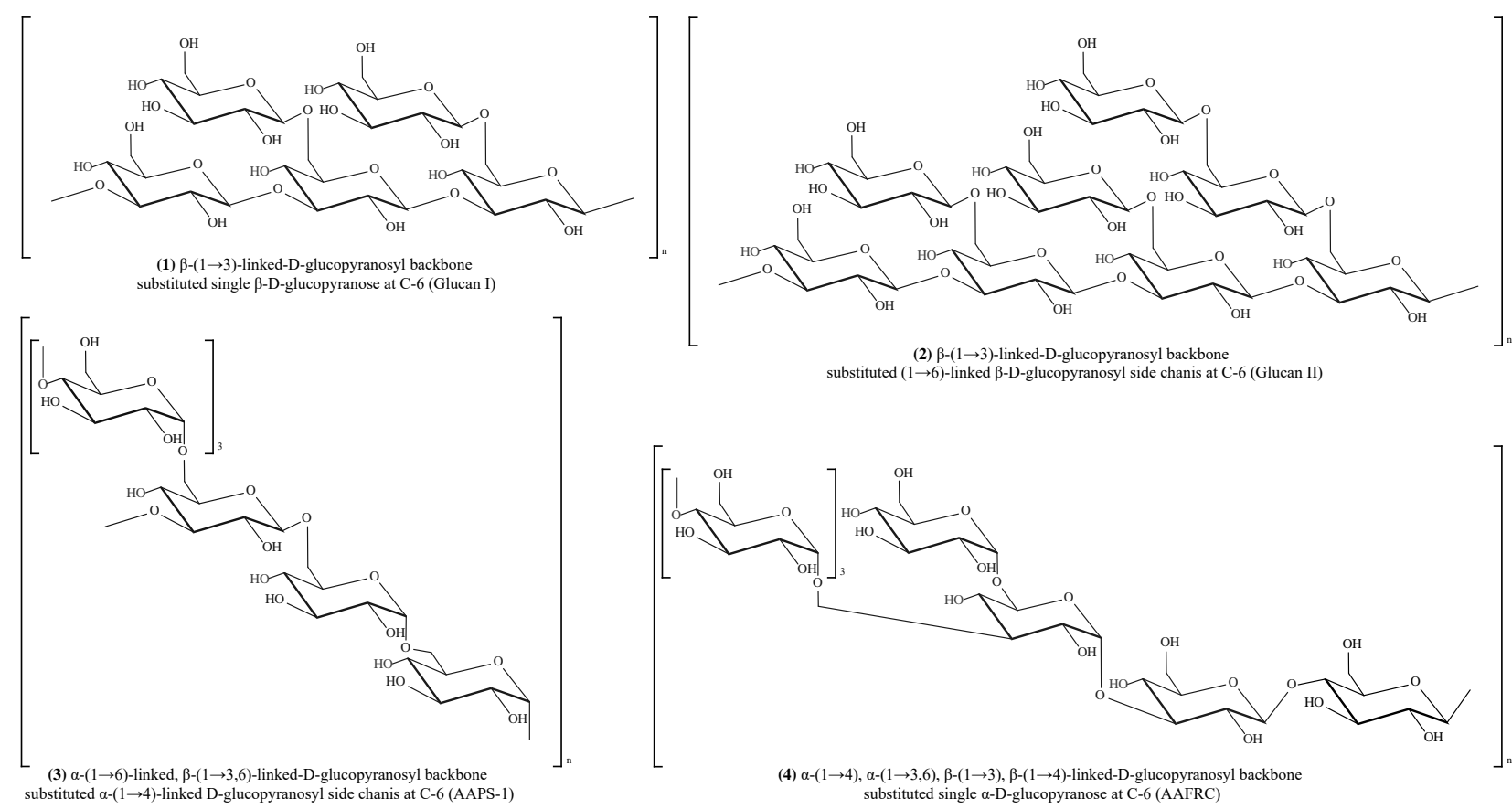

Figure 2 - Chemical structures of various types of homopolysaccharides from Auricularia spp. 1 Glucan I. 2 Glucan II (Sone et al. 1978, Misaki et al. 1981, Misaki \& Kakuta 1995). 3 AAPS-1, 4 AAFRC (Song \& Du 2012a, b).

\section{Polysaccharides of Auricularia}

Polysaccharides are considered to be the most potent group of bioactive compounds found in mushrooms (Friedman 2016, Singdevsachan et al. 2016, Sánchez 2017), serving as the structural components of the cell wall (Zhang et al. 2007, Dalonso et al. 2015) and as carbohydrate reserves in sclerotia (Stone 2009). Polysaccharides seem to be the major constituents of Auricularia and are 
classified as homopolysaccharides or heteropolysaccharides based on their monomeric composition (Sone et al. 1978, Misaki et al. 1981). Homopolysaccharides, composed of $\beta$ - $(1 \rightarrow 3)$-linked D-glucopyranosyl backbone substituted $(1 \rightarrow 6)$-linked $\beta$-D-glucopyranosyl groups, were the most abundantly found macromolecules in most of the edible, medicinal mushrooms (Sone et al. 1978, Misaki \& Kakuta 1995, Dalonso et al. 2015, Friedman 2016), including Auricularia species such as A. auricula-judae (Sone et al. 1978, Misaki et al. 1981, Misaki \& Kakuta 1995), A. polytricha (Song \& Du 2010), and Auricularia sp. (Yū ěr) (Kiho et al. 1987, 1991). Glucan I (Fig. 2.1) and Glucan II (Fig. 2.2) homopolysaccharides were

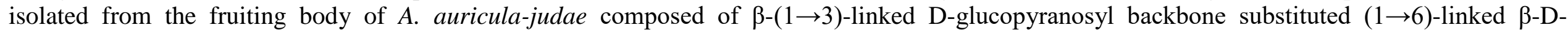
glucopyranosyl groups (Sone et al. 1978, Misaki et al. 1981, Misaki \& Kakuta 1995). Homopolysaccharides composed of different conformations and different linkages of D-glucopyranosyl backbones were reported from A. polytricha AAPS-1 (Fig. 2.3) (Song \& Du 2012b), AAFRC (Fig. 2.4) (Song \& Du 2012a). Different numbers of $(1 \rightarrow 6)$-D-glycosidic linkages attached to the $\beta$ - $(1 \rightarrow 3)$-linked D-glucopyranosyl backbones of Auricularia polysaccharides were attributed to various chemical, physical and bioactive properties (Table 4).

Table 4 Structure elucidated polysaccharides of different Auricularia species: extraction process, chemical, physical and pharmacological properties.

\begin{tabular}{|c|c|c|c|c|c|c|c|}
\hline $\begin{array}{l}\text { Auricularia } \\
\text { species }\end{array}$ & Extraction & Chemical structure & $\begin{array}{l}\text { Chemical and physical } \\
\text { properties }\end{array}$ & $\begin{array}{l}\text { Pharmacological } \\
\text { properties, Tested } \\
\text { living system }\end{array}$ & $\begin{array}{l}\text { Routes of } \\
\text { administrat } \\
\text { ion, Dose }\end{array}$ & $\begin{array}{l}\text { Inhibition/ } \\
\text { Decreasing } \\
\text { ratio }\end{array}$ & References \\
\hline $\begin{array}{l}\text { Auricularia } \\
\text { auricula* }^{*}\end{array}$ & $\begin{array}{l}\text { Dried fruit body } \rightarrow \text { hot water } \\
\text { extraction }\left(100^{\circ} \mathrm{C} / 2 \text { times } / 4 \mathrm{~h}\right) \rightarrow \\
\text { supernatant } \rightarrow \text { precipitated with } \\
\text { EtOH } \rightarrow \text { AAP } \rightarrow \\
\text { carboxymethylation } \rightarrow \text { CMAAP } \\
\rightarrow \text { purified } \rightarrow \text { CMAAP } 22^{* *} \\
\end{array}$ & $\begin{array}{l}\text { CMAAP22: } \beta-(1 \rightarrow 3) \text {-linked } \\
\text { D- mannopyranosyl } \\
\text { backbone substituted with } \\
\beta \text {-D-glucose and partially } \\
\text { substituted with }-\mathrm{CH}_{2} \mathrm{COOH} \\
\text { at C-2, C-4, C-6 }\end{array}$ & $\begin{array}{l}\text { mannose:glucose }= \\
\text { 1.06:1. m.w. } 3.4 \times 10^{6} \\
\text { Da. }\end{array}$ & $\begin{array}{l}\text { Antioxidant activity } \\
\text { in vitro: } \mathrm{OH}^{-}, \mathrm{ABTS}^{+} \\
\text {radical scavenging } \\
\text { activity }\end{array}$ & $\begin{array}{l}2 \mathrm{mg} / \mathrm{ml} \\
\text { (r.s.: vitamin } \\
\text { C) }\end{array}$ & $\begin{array}{l}\text { Scavenging } \\
\text { rate } 52.1 \% \text {, } \\
\approx 70 \%\end{array}$ & $\begin{array}{l}\text { (Yang et al. } \\
2011 \text { ) }\end{array}$ \\
\hline $\begin{array}{l}\text { A. auricula- } \\
\text { judae* }\end{array}$ & $\begin{array}{l}\text { Dried fruit body } \rightarrow 0.9 \% \mathrm{NaCl}_{(\mathrm{aq})} \\
(24 \mathrm{~h}) \rightarrow \text { Residue } \rightarrow \text { hot water } \\
\text { extract }\left(120^{\circ} \mathrm{C} / 20 \text { min. }\right) \rightarrow \\
\text { precipitated with Cetylpyridinium } \\
\text { chloride }(\mathrm{CPC}) \rightarrow \text { supernatant } \rightarrow \\
\text { precipitated with EtOH } \rightarrow \text { Glucan } \\
\text { I }\end{array}$ & 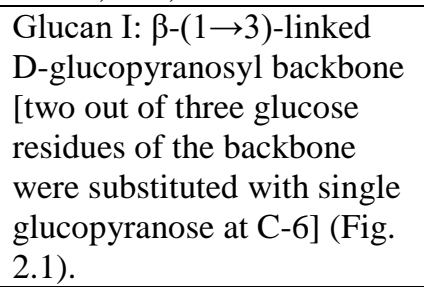 & $\begin{array}{l}{[\alpha]_{\mathrm{D}}^{25}-10.1^{\circ}(c=1.5,} \\
0.5 \mathrm{M} \mathrm{NaOH}) . \text { Neutral, } \\
\text { water-soluble. } 2.3 \% \\
\text { yield. m.w. } 1.4 \times 10^{6}\end{array}$ & $\begin{array}{l}\text { Anti-tumor activity } \\
\text { on sarcoma } 180 \text { cells } \\
\text { implanted ICR-JCR } \\
\text { mice }\end{array}$ & $\begin{array}{l}\text { i.p., } 8 \mathrm{mg} \times \\
10 \text { days }\end{array}$ & $\begin{array}{l}\text { Average } \\
\text { tumor } \\
\text { weight } \\
96.6 \%\end{array}$ & $\begin{array}{l}\text { Sone et al. } \\
1978, \\
\text { Misaki et al. } \\
1981, \\
\text { Misaki \& } \\
\text { Kakuta } \\
\text { 1995) }\end{array}$ \\
\hline $\begin{array}{l}\text { A. auricula- } \\
\text { judae* }\end{array}$ & $\begin{array}{l}\text { Dried fruit body } \rightarrow 0.9 \% \mathrm{NaCl}_{(\mathrm{aq})} \\
(24 \mathrm{~h}) \rightarrow \text { residue } \rightarrow \text { hot water } \\
\text { extraction }\left(120^{\circ} \mathrm{C} / 20 \text { min. }\right) \rightarrow \\
\text { residue } \rightarrow \text { hot alkali extraction } \\
\left(1 \mathrm{M} \mathrm{NaOH} / 65^{\circ} \mathrm{C} / 2 \mathrm{~h}\right) \rightarrow \text { residue } \\
\rightarrow \text { Glucan II }\end{array}$ & $\begin{array}{l}\text { Glucan II: } \beta \text { - }(1 \rightarrow 3) \text {-linked } \\
\text { D-glucopyranosyl backbone } \\
\text { [three out of four glucose } \\
\text { residues of the backbone } \\
\text { were substituted with single } \\
\text { glucopyranose units, and } \\
(1 \rightarrow 6)-D \text { glucosidic linked } \\
\text { short side chains (very few) } \\
\text { at C-6] (Fig. } 2.2 \text { ). }\end{array}$ & $\begin{array}{l}\text { Water, alkali }(2 \mathrm{M} \\
\mathrm{NaOH}) \text { or } \mathrm{Me}_{2} \mathrm{SO} \\
\text { insoluble. } 49 \% \text { yield. } \\
\text { m.w. } \sim 6 \times 10^{3}\end{array}$ & $\begin{array}{l}\text { Anti-tumor activity } \\
\text { on sarcoma } 180 \text { cells } \\
\text { implanted ICR-JCR } \\
\text { mice }\end{array}$ & $\begin{array}{l}\text { i.p., } 10 \mathrm{mg} \times \\
10 \text { days }\end{array}$ & $\begin{array}{l}\text { Average } \\
\text { tumor } \\
\text { weight } \\
18.9 \%\end{array}$ & $\begin{array}{l}\text { (Sone et al. } \\
1978, \\
\text { Misaki et al. } \\
1981, \\
\text { Misaki \& } \\
\text { Kakuta } \\
\text { 1995) }\end{array}$ \\
\hline
\end{tabular}


Table 4 Continued.

\begin{tabular}{|c|c|c|c|c|c|c|c|}
\hline $\begin{array}{l}\text { Auricularia } \\
\text { species }\end{array}$ & Extraction & Chemical structure & $\begin{array}{l}\text { Chemical and physical } \\
\text { properties }\end{array}$ & $\begin{array}{l}\text { Pharmacological } \\
\text { properties, Tested } \\
\text { living system }\end{array}$ & $\begin{array}{l}\text { Routes of } \\
\text { administrat } \\
\text { ion, Dose } \\
\end{array}$ & $\begin{array}{l}\text { Inhibition/ } \\
\text { Decreasing } \\
\text { ratio } \\
\end{array}$ & References \\
\hline $\begin{array}{l}\text { A. auricula- } \\
\text { judae }\end{array}$ & $\begin{array}{l}\text { Dried fruit body } \rightarrow 0.9 \% \mathrm{NaCl}_{(\mathrm{aq})} \\
(24 \mathrm{~h}) \rightarrow \text { Residue } \rightarrow \text { hot water } \\
\text { extraction }\left(120^{\circ} \mathrm{C} / 20 \text { min. }\right) \rightarrow \\
\text { precipitated with Cetylpyridinium } \\
\text { chloride } \rightarrow \text { dissolve } 10 \% \\
\mathrm{NaCl}_{(\mathrm{aq})} \rightarrow \text { precipitated with } \\
\mathrm{EtOH} \rightarrow \text { heteropolysaccharide }\end{array}$ & \multirow{2}{*}{ 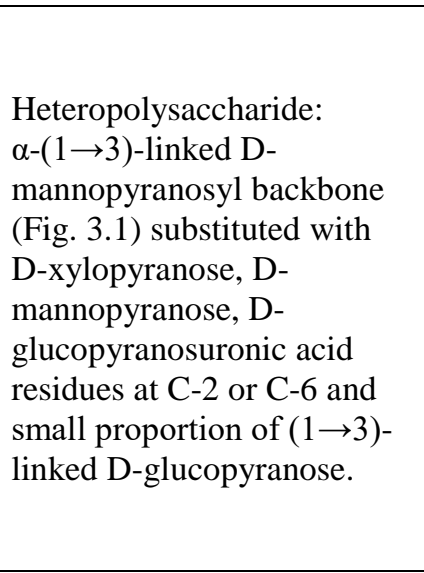 } & $\begin{array}{l}{[\alpha]_{\mathrm{D}}^{25}-20.3^{\circ}(0.5 \mathrm{M}} \\
\text { NaOH). Water-soluble. } \\
\text { Molar ratio of D-xylose: } \\
\text { D-mannose: D-glucose: } \\
\text { D-glucuronic acid = 1: } \\
\text { 4.1: } 1.3: 1.3 .48 .7 \% \\
\text { yield. m.w. } 5 \times 10^{5}\end{array}$ & $\begin{array}{l}\text { Anti-tumor activity } \\
\text { on sarcoma } 180 \text { cells } \\
\text { implanted ICR-JCR } \\
\text { mice }\end{array}$ & $\begin{array}{l}\text { i.p., } 10 \mathrm{mg} \times \\
10 \text { days }\end{array}$ & $\begin{array}{l}\text { Average } \\
\text { tumor } \\
\text { weight } \\
18.5 \%\end{array}$ & $\begin{array}{l}\text { (Sone et al. } \\
1978, \\
\text { Misaki et al. } \\
1981 \text { ) }\end{array}$ \\
\hline $\begin{array}{l}\text { A. auricula- } \\
\text { judae }\end{array}$ & $\begin{array}{l}\text { Dried fruit body } \rightarrow \mathrm{NaCl}_{(\mathrm{aq})} \\
(24 \mathrm{~h}) \rightarrow \text { Residue } \rightarrow \text { hot water } \\
\text { extraction }\left(120^{\circ} \mathrm{C} / 20 \mathrm{~min} .\right) \rightarrow \\
\text { residue } \rightarrow \text { hot alkali }(1 \mathrm{M} \\
\left.\mathrm{NaOH} / 65^{\circ} \mathrm{C} / 2 \mathrm{~h}\right) \text { extract } \rightarrow \\
\text { precipitated with EtOH } \rightarrow \\
\text { heteropolysaccharide }\end{array}$ & & $\begin{array}{l}\text { Alkali soluble. Molar } \\
\text { ratio of D-xylose: D- } \\
\text { mannose: D-glucose: D- } \\
\text { glucuronic acid = 1: } 2.1 \text { : } \\
\text { 1: } 0.6 \text {. }\end{array}$ & - & - & - & $\begin{array}{l}\text { (Sone et al. } \\
1978 \text { ) }\end{array}$ \\
\hline \multirow[t]{2}{*}{$\begin{array}{l}\text { A. auricula- } \\
\text { judae }\end{array}$} & \multirow{2}{*}{$\begin{array}{l}\text { Dried fruit body } \rightarrow \text { hot } \mathrm{MeOH} \\
\text { extraction } \rightarrow \text { residue } \rightarrow \text { hot } 70 \% \\
\text { EtOH extract }(3 \text { times } / 20 \mathrm{~h}) \rightarrow \\
\text { purified then fractionated by } \\
\text { cetyltrimethylammonium bromide } \\
(\text { CTAB }) \rightarrow \text { MEA** }^{* *}\end{array}$} & \multirow{4}{*}{$\begin{array}{l}\text { MEA and MHA } \\
\text { (heteropolysaccharide): } \alpha \text { - } \\
(1 \rightarrow 3 \text { )-linked D- } \\
\text { mannopyranosyl backbone } \\
\text { (Fig. 3.1) substituted with } \beta \text { - } \\
\text { D- glucopyranosyluronic } \\
\text { acid at C-2, single residue or } \\
\text { short chains of } \beta \text {-D- } \\
\text { xylopyranose at C-2, C-6 } \\
\text { and small amount of }(1 \rightarrow 6) \text { - } \\
\text { linked D- mannopyranose } \\
\text { (and/or D-glucopyranose), } \\
\text { branching D- } \\
\text { mannopyranose at C-4, C- } \\
6 \text {. }\end{array}$} & \multirow{2}{*}{$\begin{array}{l}{[\alpha]_{\mathrm{D}}^{2 \pi}+31^{\circ}\left(c=1, \mathrm{H}_{2} \mathrm{O}\right) .} \\
\mathrm{Me} 2 \mathrm{SO} \text { slightly soluble. } \\
\text { Molar ratio D-glucuronic } \\
\text { acid: D-xylose: D- } \\
\text { mannose = 1: } 0.5: 2.8 . \\
\text { 4\% yield. m.w. } 3.7 \times 10^{5}\end{array}$} & $\begin{array}{l}\text { Anti-tumor activity } \\
\text { on sarcoma } 180 \text { cells } \\
\text { implanted ddY male } \\
\text { mice }\end{array}$ & $\begin{array}{l}\text { i.p., } 25 \mathrm{mg} \times \\
10 \text { days }\end{array}$ & $\begin{array}{l}\text { Average } \\
\text { tumor } \\
\text { weight } \\
42 \% \\
\end{array}$ & $\begin{array}{l}\text { (Ukai et al. } \\
1982, \\
1983 b)\end{array}$ \\
\hline & & & & $\begin{array}{l}\text { Anti-inflammatory } \\
\text { activity on Sprague } \\
\text { Dawley mice: } \\
\text { Carrageenin edema, } \\
\text { Scald hyperalgesia }\end{array}$ & $\begin{array}{l}\text { i.p., } 50 \\
\mathrm{mg} / \mathrm{kg} \times 2 \\
\text { times } \\
\text { immediately } \\
\text { and 1h after }\end{array}$ & $\begin{array}{l}\text { Hind paw } \\
\text { thickness } \\
0 \% \text {, Pain } \\
\text { threshold } \\
6 \%\end{array}$ & $\begin{array}{l}\text { (Ukai et al. } \\
\text { 1983a) }\end{array}$ \\
\hline \multirow[t]{2}{*}{$\begin{array}{l}\text { A. auricula- } \\
\text { judae }\end{array}$} & \multirow{2}{*}{$\begin{array}{l}\text { Dried fruit body } \rightarrow \text { hot } \mathrm{MeOH} \\
\text { extraction } \rightarrow \text { Residue } \rightarrow \text { Hot } \\
70 \% \text { EtOH extraction }(3 \\
\text { times } / 20 \mathrm{~h}) \rightarrow \text { residue } \rightarrow \text { hot } \\
\text { water extract }\left(93-98^{\circ} \mathrm{C} / 4\right. \\
\text { times } / 4 \mathrm{~h}) \rightarrow \text { purified then } \\
\text { fractionated by CTAB } \rightarrow \mathrm{MHA}^{* *}\end{array}$} & & \multirow{2}{*}{$\begin{array}{l}{[\alpha]_{\mathrm{D}}^{2 \mathrm{a}}+33^{\circ}\left(c=1, \mathrm{H}_{2} \mathrm{O}\right) .} \\
\mathrm{Me}_{2} \mathrm{SO} \text { insoluble. Molar } \\
\text { ratio D-glucuronic acid: } \\
\text { D-xylose: D-mannose = } \\
\text { 1: } 0.6: 1.16 \% \text { yield. } \\
\text { m.w. } 3 \times 10^{5}\end{array}$} & $\begin{array}{l}\text { Anti-tumor activity } \\
\text { on sarcoma } 180 \text { cells } \\
\text { implanted ddY male } \\
\text { mice }\end{array}$ & $\begin{array}{l}\text { i.p., } 25 \mathrm{mg} \times \\
10 \text { days }\end{array}$ & $\begin{array}{l}\text { Average } \\
\text { tumor } \\
\text { weight } \\
29 \% \\
\end{array}$ & $\begin{array}{l}\text { (Ukai et al. } \\
1982, \\
1983 b)\end{array}$ \\
\hline & & & & $\begin{array}{l}\text { Anti-inflammatory } \\
\text { activity on Sprague } \\
\text { Dawley mice: } \\
\text { Carrageenan induced } \\
\text { edema, Scald induced } \\
\text { hyperalgesia }\end{array}$ & $\begin{array}{l}\text { i.p., } 50 \\
\mathrm{mg} / \mathrm{kg} \times 2 \\
\text { times } \\
\text { immediately } \\
\text { and 1h after }\end{array}$ & $\begin{array}{l}\text { Hind paw } \\
\text { thickness } \\
33 \% \text {, Pain } \\
\text { threshold } \\
19 \%\end{array}$ & $\begin{array}{l}\text { (Ukai et al. } \\
\text { 1983a) }\end{array}$ \\
\hline
\end{tabular}


Table 4 Continued.

\begin{tabular}{|c|c|c|c|c|c|c|c|}
\hline $\begin{array}{l}\text { Auricularia } \\
\text { species }\end{array}$ & Extraction & Chemical structure & $\begin{array}{l}\text { Chemical and physical } \\
\text { properties }\end{array}$ & $\begin{array}{l}\text { Pharmacological } \\
\text { properties, Tested } \\
\text { living system }\end{array}$ & $\begin{array}{l}\text { Routes of } \\
\text { administrat } \\
\text { ion, Dose }\end{array}$ & $\begin{array}{l}\text { Inhibition/ } \\
\text { Decreasing } \\
\text { ratio }\end{array}$ & References \\
\hline $\begin{array}{l}\text { A. auricula- } \\
\text { judae* }\end{array}$ & $\begin{array}{l}\text { Dried fruit body } \rightarrow \text { hot ethyl } \\
\text { acetate and methanol } \rightarrow \text { Residue } \\
\rightarrow 70 \% \text { ethanol/water extract }\end{array}$ & 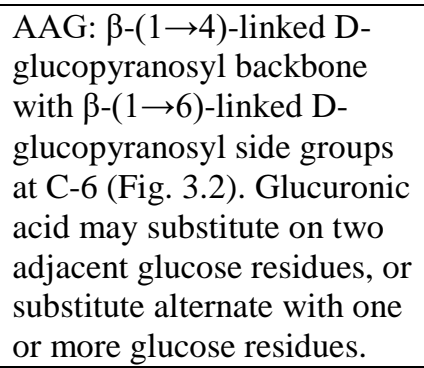 & $\begin{array}{l}\text { Water soluble, D- } \\
\text { glucose: D-glucuronic } \\
\text { acid = 6: } 1 \text {. m.w. } 2.88 \times \\
10^{5}\end{array}$ & $\begin{array}{l}\text { Anti-tumor activity } \\
\text { on acinar cell } \\
\text { carcinoma in vitro, } \\
\text { sarcoma } 180 \text { cells } \\
\text { implanted BALB/c } \\
\text { male mice }\end{array}$ & $\begin{array}{l}\text { (1) } 0.05 \\
\mathrm{mg} / \mathrm{l}(2) \text { i.p., } \\
20 \mathrm{mg} / \mathrm{kg} \times 8 \\
\text { days. (r.s.: } \\
\text { 5- } \\
\text { Fluorouracil } \\
\text { ) }\end{array}$ & $\begin{array}{l}\text { Average } \\
\text { tumor } \\
\text { weight } \\
34.1 \% \text {, } \\
39.1 \%\end{array}$ & $\begin{array}{l}\text { (Ma et al. } \\
2008,2010)\end{array}$ \\
\hline $\begin{array}{l}\text { A. } \\
\text { polytricha* }\end{array}$ & $\begin{array}{l}\text { Dried fruit body } \rightarrow \text { hot water } \\
\text { extract }\left(90^{\circ} \mathrm{C} / 3 \text { times } / 3 \mathrm{~h}\right) \rightarrow \\
\text { precipitated with } \mathrm{EtOH} \rightarrow \\
\text { purified } \rightarrow \text { APPS } \rightarrow \text { HSCCC } \\
\text { separation } \rightarrow \text { AAPS-1, AAPS-2, } \\
\text { AAPS-3** }\end{array}$ & $\begin{array}{l}\text { AAPS-2: } \beta \text { - }(1 \rightarrow 3) \text {-linked } \\
\text { D-glucopyranosyl backbone } \\
\text { [one out of three glucose } \\
\text { residues of the backbone } \\
\text { were substituted with single } \\
\beta \text {-D-glucopyranose at C-6] }\end{array}$ & m.w. $2.59 \times 10^{5} \mathrm{Da}$ & $\begin{array}{l}\text { Anti-tumor activity } \\
\text { on sarcoma } 180 \text { cells } \\
\text { implanted Swiss } \\
\text { albino female mice }\end{array}$ & $\begin{array}{l}\text { p.o., } 12 \\
\text { mg/kg/day } \\
\text { (r.s.: } \\
\text { cyclophos- } \\
\text { phamide) }\end{array}$ & $\begin{array}{l}\text { Average } \\
\text { tumor } \\
\text { weight } \\
40.43 \%\end{array}$ & $\begin{array}{l}\text { (Song \& Du } \\
\text { 2010) }\end{array}$ \\
\hline $\begin{array}{l}\text { A. } \\
\text { polytricha* }\end{array}$ & $\begin{array}{l}\text { Dried fruit body } \rightarrow \text { hot water } \\
\text { extract }\left(90^{\circ} \mathrm{C} / 3 \text { times } / 3 \mathrm{~h}\right) \rightarrow \\
\text { precipitated with EtOH } \rightarrow \\
\text { purified } \rightarrow \text { APPS } \rightarrow \text { HSCCC } \\
\text { separation } \rightarrow \text { AAPS- } 1^{* *}\end{array}$ & $\begin{array}{l}\text { AAPS-1: } \alpha-(1 \rightarrow 6), \beta- \\
(1 \rightarrow 6)-\text {-linked D- } \\
\text { glucopyranosyl backbone } \\
\text { substituted with } \alpha-(1 \rightarrow 4)- \\
\text { linked D-glucopyranose } \\
\text { side chains (Fig. } 2.3)\end{array}$ & $\begin{array}{l}\text { Water-soluble. m.w. } 1.62 \\
\times 10^{5} \mathrm{Da}\end{array}$ & - & - & - & $\begin{array}{l}\text { (Song \& Du } \\
\text { 2012b) }\end{array}$ \\
\hline $\begin{array}{l}\text { A. } \\
\text { polytricha* }\end{array}$ & $\begin{array}{l}\text { Dried fruit body } \rightarrow \text { hot water } \\
\text { extract }\left(100^{\circ} \mathrm{C} / 2 \mathrm{~h}\right) \rightarrow \text { centrifuged } \\
\text { residual } \rightarrow \text { hot } 0.9 \% \text { NaCl extract } \\
\left(100^{\circ} \mathrm{C} / 2 \mathrm{~h}\right) \rightarrow \text { centrifuged } \\
\text { residual } \rightarrow 0.05 \mathrm{M} \mathrm{NaOH} \text { extract } \\
\left(50^{\circ} \mathrm{C} / 2 \mathrm{~h}\right) \rightarrow \text { AAFRC** }\end{array}$ & $\begin{array}{l}\text { AAFRC: } \alpha-(1 \rightarrow 3), \alpha- \\
(1 \rightarrow 4), \beta-(1 \rightarrow 3), \beta-(1 \rightarrow 4)- \\
\text { linked D-glucopyranosyl } \\
\text { backbone substituted with } \\
\text { single } \alpha-(1 \rightarrow 6) \text {-linked D- } \\
\text { glucopyranose at C-6 on } \\
\text { every six residues (Fig. } 2.4 \text { ) }\end{array}$ & $\begin{array}{l}\text { Water-soluble. m.w. } 1.2 \\
\times 10^{6} \mathrm{Da}\end{array}$ & $\begin{array}{l}\text { Anti-tumor activity } \\
\text { on sarcoma } 180 \text { cells } \\
\text { implanted ICR } \\
\text { female mice }\end{array}$ & $\begin{array}{l}\text { i.p., } 24 \\
\mathrm{mg} / \mathrm{kg} / \mathrm{day} \\
\text { (r.s.: } \\
\text { cyclophos- } \\
\text { phamide) }\end{array}$ & $\begin{array}{l}\text { Average } \\
\text { tumor } \\
\text { weight } \\
43.61 \%\end{array}$ & $\begin{array}{l}\text { (Song \& Du } \\
\text { 2012a) }\end{array}$ \\
\hline $\begin{array}{l}\text { Auricularia } \\
\text { sp.* (Yū } \\
\text { ěr) }\end{array}$ & $\begin{array}{l}\text { Dried fruit body } \rightarrow \text { extracted with } \\
\text { hot } \mathrm{MeOH} \text {, hot } 70 \% \text { EtOH, hot } \\
\text { water Successively } \rightarrow \text { residue } \\
\text { extracted with } \mathrm{NaOH}\left(1 \mathrm{M} / 25^{\circ} \mathrm{C}\right) \\
\rightarrow \text { precipitated with EtOH } \rightarrow \text { N- } \\
5 \mathrm{P}^{* *}\end{array}$ & $\begin{array}{l}\text { N-5P: } \quad \beta-(1 \rightarrow 3) \text {-linked D- } \\
\text { glucosyl backbone } \\
\text { substituted with single } \\
\text { glucose units at C-6. }\end{array}$ & $\begin{array}{l}{[\alpha]_{\mathrm{D}}^{2 \mathrm{~d}}+2.3^{\circ}(c=0.51,} \\
0.5 \mathrm{M} \mathrm{NaOH}) . \text { Water, } \\
\text { acidic insoluble. Alkali, } \\
\mathrm{Me}_{2} \mathrm{SO} \text { soluble. } 1.2 \% \\
\text { yield. m.w. } 5.6 \times 10^{5}\end{array}$ & $\begin{array}{l}\text { Anti-tumor activity } \\
\text { on sarcoma } 180 \text { cells } \\
\text { implanted ddY male } \\
\text { mice }\end{array}$ & $\begin{array}{l}\text { i.p., } 10 \\
\mathrm{mg} / \mathrm{kg} \times 10 \\
\text { days }\end{array}$ & $\begin{array}{l}\text { Average } \\
\text { tumor } \\
\text { weight } \\
81 \%\end{array}$ & $\begin{array}{l}\text { (Kiho et al. } \\
1987 \text { ) }\end{array}$ \\
\hline
\end{tabular}


Table 4 Continued.

\begin{tabular}{|c|c|c|c|c|c|c|c|}
\hline $\begin{array}{l}\text { Auricularia } \\
\text { species }\end{array}$ & Extraction & Chemical structure & $\begin{array}{l}\text { Chemical and physical } \\
\text { properties }\end{array}$ & $\begin{array}{l}\text { Pharmacological } \\
\text { properties, Tested } \\
\text { living system }\end{array}$ & $\begin{array}{l}\text { Routes of } \\
\text { administrat } \\
\text { ion, Dose }\end{array}$ & $\begin{array}{l}\text { Inhibition/ } \\
\text { Decreasing } \\
\text { ratio }\end{array}$ & References \\
\hline $\begin{array}{l}\text { Auricularia } \\
\text { sp.* (Yū ěr) }\end{array}$ & $\begin{array}{l}\text { Dried fruit body } \rightarrow \text { hot } \mathrm{MeOH} \\
\text { extract }+ \text { residue extracted with } \\
\text { hot } 70 \% \text { EtOH }+ \text { residue } \\
\text { extracted with hot water ( } 4 \\
\text { times } / 5 \mathrm{~h}) \rightarrow \text { Purified, precipitated } \\
\text { with EtOH } \rightarrow \text { U-3-EP } \rightarrow \\
\text { fractionated by CTAB } \rightarrow \text { U-3-A } \\
\text { precipitated } \rightarrow \text { supernatant } \rightarrow \\
\text { purified } \rightarrow \text { U-3-N** }\end{array}$ & $\begin{array}{l}\text { U-3-N: } \beta \text { - }(1 \rightarrow 3) \text {-linked D- } \\
\text { glucosyl backbone } \\
\text { substituted with single } \beta \text { - } \\
(1 \rightarrow 6) \text {-linked D-glucose. }\end{array}$ & $\begin{array}{l}{[\alpha]_{\mathrm{D}}^{2 \mathrm{~d}}+1.0^{\circ}(c=0.13,} \\
0.5 \mathrm{M} \mathrm{NaOH}) . \text { Water } \\
\text { insoluble. Alkali soluble. } \\
1.24 \% \text { yield. m.w. } 6.1 \times \\
10^{5}\end{array}$ & $\begin{array}{l}\text { Anti-tumor activity } \\
\text { on sarcoma } 180 \text { cells } \\
\text { implanted ddY male } \\
\text { mice }\end{array}$ & $\begin{array}{l}\text { i.p., } 1 \mathrm{mg} / \mathrm{kg} \\
\times 10 \text { days }\end{array}$ & $\begin{array}{l}\text { Average } \\
\text { tumor } \\
\text { weight } \\
84 \%\end{array}$ & $\begin{array}{l}\text { (Kiho et al. } \\
\text { 1991) }\end{array}$ \\
\hline $\begin{array}{l}\text { Auricularia } \\
\text { sp.* (Yū ěr) }\end{array}$ & $\begin{array}{l}\text { Dried fruit body } \rightarrow \text { hot } \mathrm{MeOH} \\
\text { extract }+ \text { residue extracted with } \\
\text { hot } 70 \% \text { EtOH }+ \text { residue } \\
\text { extracted with hot water ( } 4 \\
\text { times } / 5 \mathrm{~h}) \rightarrow \text { purified, precipitated } \\
\text { with EtOH } \rightarrow \text { U-3-EP } \rightarrow \\
\text { fractionated by CTAB } \rightarrow \text { U-3-A } \\
\text { precipitated } \rightarrow \text { supernatant } \rightarrow \\
\text { purified } \rightarrow \text { fractionated eluted by } \\
\mathrm{NaCl} \rightarrow \mathrm{U}-3-\mathrm{AP}_{1} * *\end{array}$ & $\begin{array}{l}\text { U-3-AP } 1 \text { : } \beta \text { - }(1 \rightarrow 3) \text {-linked } \\
\text { D-glucosyl backbone } \\
\text { substituted with } \beta \text { - }(1 \rightarrow 6)- \\
\text { linked D-glucosyl residue. }\end{array}$ & $\begin{array}{l}{[\alpha]_{\mathrm{D}}^{2 \mathrm{a}}+2.5^{\circ}(c=0.13,1 \mathrm{M}} \\
\mathrm{NaOH}) . \text { Water insoluble. } \\
\text { Alkali soluble. } 0.63 \% \\
\text { yield. m.w. } 6.4 \times 10^{4}\end{array}$ & $\begin{array}{l}\text { Anti-tumor activity } \\
\text { on sarcoma } 180 \text { cells } \\
\text { implanted ddY male } \\
\text { mice }\end{array}$ & $\begin{array}{l}\text { i.p., } 1 \mathrm{mg} / \mathrm{kg} \\
\times 10 \text { days }\end{array}$ & $\begin{array}{l}\text { Average } \\
\text { tumor } \\
\text { weight } 8 \%\end{array}$ & $\begin{array}{l}\text { (Kiho et al. } \\
\text { 1991) }\end{array}$ \\
\hline
\end{tabular}

m.w., molecular weight; r.s., reference standard; p.o., oral administration; s.c., subcutaneous injection.

Table 5 Polysaccharides with unknown structure of different Auricularia species: extraction process and pharmacological properties.

\begin{tabular}{|c|c|c|c|c|c|}
\hline $\begin{array}{l}\text { Auricularia } \\
\text { species }\end{array}$ & Extraction & $\begin{array}{l}\text { Pharmacological activities / } \\
\text { Tested living system }\end{array}$ & $\begin{array}{l}\text { Routes of } \\
\text { administration/ } \\
\text { Dose }\end{array}$ & Inhibition / Decreasing ratio & References \\
\hline $\begin{array}{l}\text { Auricularia } \\
\text { auricula* }^{*}\end{array}$ & $\begin{array}{l}\text { Dried fruit body } \rightarrow \text { refluxed with EtOH } \\
\left(76^{\circ} \mathrm{C} / 2 \mathrm{~h} / 2 \text { times }\right) \rightarrow \text { suspension } \rightarrow \text { purified } \\
\rightarrow \text { supernatant } \rightarrow \text { extracted with EtOH } \\
\left(4^{\circ} \mathrm{C} / 24 \mathrm{~h}\right) \rightarrow \text { concentrated and lyophilized } \rightarrow \\
\text { crude polysaccharide }\end{array}$ & $\begin{array}{l}\text { Anti-coagulant activity on } \\
\text { Sprague-Dawley male rats in } \\
\text { ex vivo }\end{array}$ & $\begin{array}{l}\text { p.o., } 300 \mathrm{mg} / \mathrm{kg} \\
\text { body weight/ day } \\
\times 4 \text { weeks (r.s.: } \\
\text { aspirin) }\end{array}$ & $\begin{array}{l}\text { Inhibitory effects on platelet } \\
\text { aggregation }=39.6 \%\end{array}$ & $\begin{array}{l}\text { (Yoon et al. } \\
\text { 2003) }\end{array}$ \\
\hline
\end{tabular}


Table 5 Continued.

\begin{tabular}{|c|c|c|c|c|c|}
\hline $\begin{array}{l}\text { Auricularia } \\
\text { species }\end{array}$ & Extraction & $\begin{array}{l}\text { Pharmacological activities / } \\
\text { Tested living system }\end{array}$ & $\begin{array}{l}\text { Routes of } \\
\text { administration/ } \\
\text { Dose }\end{array}$ & Inhibition / Decreasing ratio & References \\
\hline A. auricular & $\begin{array}{l}\text { Fresh fruit body } \rightarrow \text { hot water extract } \\
\left(100^{\circ} \mathrm{C} / 2 \mathrm{~h}\right) \rightarrow \text { centrifuged } \rightarrow \text { supernatant } \rightarrow \\
\text { purified } \rightarrow \text { precipitated with EtOH } \rightarrow \\
\text { dialyzed and purified } \rightarrow \text { CAAP** }\end{array}$ & $\begin{array}{l}\text { Antioxidant activity in vitro (1) } \\
\mathrm{OH}^{-}(2) \mathrm{O}_{2}^{2-}(3) \mathrm{DPPH}(4) \mathrm{Fe}^{2+} \\
\text { radical scavenging activity }\end{array}$ & $\begin{array}{l}0-2.0 \mathrm{mg} / \mathrm{ml}(\text { r.s.: } \\
\text { vitamin } \mathrm{C})\end{array}$ & $\begin{array}{l}\mathrm{EC}_{50}=(1)>2 \mathrm{mg} / \mathrm{ml}(2)>2 \mathrm{mg} / \mathrm{ml} \\
\text { (3) } 1.62(4)>2 \mathrm{mg} / \mathrm{ml}\end{array}$ & $\begin{array}{l}\text { (He et al. } \\
\text { 2012) }\end{array}$ \\
\hline A. auricula* & $\begin{array}{l}\text { Dried fruit body } \rightarrow \text { hot water extract }(94 \\
\left.{ }^{\circ} \mathrm{C} / 3.4 \mathrm{~h}\right) \rightarrow \text { centrifuged } \rightarrow \text { supernatant } \rightarrow \\
\text { precipitated with EtOH } \rightarrow \text { centrifuged } \rightarrow \\
\text { purified } \rightarrow \text { AAFB** }\end{array}$ & $\begin{array}{l}\text { Antioxidant activity in vitro }(1) \\
\mathrm{Fe}^{2+}(2) \mathrm{OH}^{-} \text {radical scavenging } \\
\text { activity }\end{array}$ & $\begin{array}{l}0.2-1.0 \mathrm{mg} / \mathrm{ml} \\
\text { (r.s.: butylated } \\
\text { hydroxytoluene) }\end{array}$ & $\begin{array}{l}\mathrm{IC}_{50}=(1) 0.43 \mathrm{mg} / \mathrm{ml}(2) 0.38 \\
\mathrm{mg} / \mathrm{ml}\end{array}$ & $\begin{array}{l}\text { (Zou et al. } \\
\text { 2015) }\end{array}$ \\
\hline A. auricula* & $\begin{array}{l}\text { Dried fruit body } \rightarrow \text { hot water extract } \rightarrow \\
\text { precipitated with EtOH } \rightarrow \text { purified } \rightarrow \\
\text { Sulfation } \rightarrow \text { sAAPt** }\end{array}$ & $\begin{array}{l}\text { Anti-viral activity on Newcastle } \\
\text { disease virus infected SPF } \\
\text { chicken embryo in vitro }\end{array}$ & $0.244-3.91 \mu \mathrm{g} / \mathrm{ml}$ & Virus inhibitory rate $=70.90 \%$ & $\begin{array}{l}\text { (Nguyen et } \\
\text { al. 2012a) }\end{array}$ \\
\hline A. auricula & $\begin{array}{l}\text { Dried fruit body } \rightarrow \text { hot water extract }\left(100^{\circ} \mathrm{C} / 2\right. \\
\text { times } / 2 \mathrm{~h}) \rightarrow \text { precipitated with } \mathrm{EtOH} \rightarrow \\
\text { AARP** }\end{array}$ & $\begin{array}{l}\text { Hepatoprotective activity on } \\
\mathrm{CCl}_{4} \text { induced liver injured mice }\end{array}$ & $50-100 \mathrm{mg} / \mathrm{kg} / \mathrm{day}$ & $\begin{array}{l}\text { Serum aspartate aminotransferase } \\
\text { (AST) and malondialdehyde } \\
\text { aldehyde (MDA) decreased, } \\
\text { superoxide dismutase (SOD) } \\
\text { activity increased }(\mathrm{P}<0.05) \\
\end{array}$ & $\begin{array}{l}\text { (Guo et al. } \\
\text { 2015) }\end{array}$ \\
\hline A. auricula* & $\begin{array}{l}\text { Dried fruit body } \rightarrow \text { Ether extract }(\text { r.t. } / 3 \\
\text { times } / 48 \mathrm{~h}) \rightarrow \text { residue } \rightarrow \text { hot water extract } \\
\left(100^{\circ} \mathrm{C} / 2 \text { times } / 2 \mathrm{~h}\right) \rightarrow \text { precipitated with EtOH } \\
\rightarrow \text { AAP } * *\end{array}$ & $\begin{array}{l}\text { Hypocholesterolemic activity } \\
\text { on cholesterol-enriched diet fed } \\
\text { male ICR mice }\end{array}$ & $\begin{array}{l}\text { p.o., } 120 \\
\text { mg/kg/day (r.s.: } \\
\text { distilled water) }\end{array}$ & $\begin{array}{l}\text { Concentrations of total cholesterol } \\
\text { and low-density lipoprotein } \\
\text { cholesterol in blood serum } \\
\text { decreased significantly }(\mathrm{P}<0.05)\end{array}$ & $\begin{array}{l}\text { (Chen et al. } \\
\text { 2008) }\end{array}$ \\
\hline A. auricula* & $\begin{array}{l}\text { Dried fruit body } \rightarrow \text { refluxed with EtOH } \\
\left(80^{\circ} \mathrm{C} / 3 \text { times } / 2 \mathrm{~h}\right) \rightarrow \text { suspension } \rightarrow \text { extracted } \\
\text { with EtOH }\left(4^{\circ} \mathrm{C} / 24 \mathrm{~h}\right) \rightarrow \text { concentrated and } \\
\text { lyophilized } \rightarrow \mathrm{AAE}^{* *}\end{array}$ & $\begin{array}{l}\text { Hypocholesterolemic activity } \\
\text { on cholesterol-enriched diet fed } \\
\text { male ICR mice }\end{array}$ & $\begin{array}{l}\text { p.o., } 150 \\
\text { mg/kg/day (r.s.: } \\
\text { distilled water) }\end{array}$ & $\begin{array}{l}\text { Concentrations of total cholesterol } \\
\text { in blood serum and hepatic lipids } \\
\text { serum decreased significantly }(\mathrm{P}< \\
0.05)\end{array}$ & $\begin{array}{l}\text { (Chen et al. } \\
\text { 2011) }\end{array}$ \\
\hline A. auricula-judae & $\begin{array}{l}\text { Dried fruit body } \rightarrow 80 \% \text { EtOH extract } \\
\left(100^{\circ} \mathrm{C} / 2 \text { times } / 3 \mathrm{~h}\right) \rightarrow \text { residue } \rightarrow \text { suspended } \\
\text { with water } \rightarrow \text { Dichloromethane fraction }\end{array}$ & $\begin{array}{l}\text { Anti-inflammatory activity in } \\
\text { vitro on Murine macrophage } \\
\text { RAW } 264.7 \text { cells }\end{array}$ & $\begin{array}{l}100 \mu \mathrm{g} / \mathrm{ml}(\mathrm{r} . \mathrm{s} .: \\
-)\end{array}$ & $73.5 \%$ & $\begin{array}{l}\text { (Damte et al. } \\
\text { 2011) }\end{array}$ \\
\hline A. auricula-judae & $\begin{array}{l}\text { Dried fruit body } \rightarrow 95 \% \text { EtOH extract } \\
\text { (r.t./72h) }\end{array}$ & $\begin{array}{l}\text { Anti-microbial activity in vitro } \\
\text { on Bacillus subtillis, } \\
\text { Escherichia coli, Pseudomonas } \\
\text { aeruginosa, Salmonella typhi } \\
\text { and Staphylococcus aureus }\end{array}$ & $\begin{array}{l}10 \mathrm{mg} / \mathrm{ml} \text { (r.s.: } \\
\text { vancomycin) }\end{array}$ & $\begin{array}{l}\text { Minimum inhibitory } \\
\text { concentrations (mg/ml): } \\
\text { Escherichia coli, Pseudomonas } \\
\text { aeruginosa = 50; Bacillus subtillis, } \\
\text { Salmonella typhi, Staphylococcus } \\
\text { aureus }=12.5\end{array}$ & $\begin{array}{l}\text { (Deka et al. } \\
\text { 2017) }\end{array}$ \\
\hline
\end{tabular}


Table 5 Continued.

\begin{tabular}{|c|c|c|c|c|c|}
\hline $\begin{array}{l}\text { Auricularia } \\
\text { species }\end{array}$ & Extraction & $\begin{array}{l}\text { Pharmacological activities / } \\
\text { Tested living system }\end{array}$ & $\begin{array}{l}\text { Routes of } \\
\text { administration/ } \\
\text { Dose }\end{array}$ & Inhibition / Decreasing ratio & References \\
\hline A. auricula-judae & $\begin{array}{l}\text { Ethanol extract - Dichloromethane fraction } \\
\text { (Diazane + unidentified components) }\end{array}$ & $\begin{array}{l}\text { Anti-tumor activity on } \\
\text { broncheoalveolar cancer NCI } \\
\text { H538 in vitro }\end{array}$ & $\begin{array}{l}0.01-1.0 \mathrm{mg} / \mathrm{ml} \\
\text { (r.s.: doxorubicin) }\end{array}$ & $\mathrm{IC}_{50}=57.2 \mu \mathrm{g} / \mathrm{ml}$ & $\begin{array}{l}\text { (Reza et al. } \\
\text { 2014) }\end{array}$ \\
\hline A. auricula-judae & $\begin{array}{l}\text { Ethanol extract - Dichloromethane fraction } \\
\text { (Diazane + unidentified components) }\end{array}$ & $\begin{array}{l}\text { Anti-tumor activity on Gastric } \\
\text { cancer cells SNU1 in vitro }\end{array}$ & $\begin{array}{l}0.01-1.0 \mathrm{mg} / \mathrm{ml} \\
\text { (r.s.: doxorubicin) }\end{array}$ & $\mathrm{IC}_{50}=73.2 \mu \mathrm{g} / \mathrm{ml}$ & $\begin{array}{l}\text { (Reza et al. } \\
\text { 2014) }\end{array}$ \\
\hline A. auricula-judae & $\begin{array}{l}\text { Dried fruit body } \rightarrow 70 \% \text { EtOH extract } \\
\left(100^{\circ} \mathrm{C} / 6 \mathrm{~h}\right)\end{array}$ & $\begin{array}{l}\text { Anti-tumor activity in vitro on } \\
\text { (1) P388D1 macrophage, (2) } \\
\text { Sarcoma 180, (3) NCI H358 } \\
\text { (bronchoalveolar), (4) SNU1 } \\
\text { (gastric carcinoma) cells }\end{array}$ & $\begin{array}{l}1 \mathrm{mg} / \mathrm{ml}(\mathrm{r} . \mathrm{s} .: \\
\text { doxorubicin) }\end{array}$ & $\begin{array}{l}\text { (1) } 42.21 \%,(2) 65.71 \%,(3) \\
69.76 \%,(4) 68.01 \%\end{array}$ & $\begin{array}{l}\text { (Reza et al. } \\
\text { 2012) }\end{array}$ \\
\hline A. auricula-judae & Ethanol extract - Dichloromethane fraction & $\begin{array}{l}\text { Anti-tumor activity on sarcoma } \\
180 \text { tumor cells implanted } \\
\text { BALB/c mice }\end{array}$ & $\begin{array}{l}\text { p.o., } 100 \mathrm{mg} / \mathrm{kg} \times \\
10 \text { days (r.s.: } \\
\text { doxorubicin) }\end{array}$ & $42.62 \%$ & $\begin{array}{l}\text { (Reza et al. } \\
\text { 2011) }\end{array}$ \\
\hline A. auricula-judae* & $\begin{array}{l}\text { Dried fruit body } \rightarrow 85 \% \text { EtOH extract }(\text { r.t. } / 3 \\
\text { times } / 48 \mathrm{~h}) \rightarrow \text { residue } \rightarrow \text { hot water extract } \\
\left(100^{\circ} \mathrm{C} / 4 \text { times } / 3 \mathrm{~h}\right) \rightarrow \text { precipitated with } \mathrm{EtOH} \\
\rightarrow \text { polysaccharide: } \mathrm{FA}^{* *}\end{array}$ & $\begin{array}{l}\text { Hypoglycemic activity on male } \\
\text { KK-A }{ }^{y} \text { genetically diabetic } \\
\text { mice }\end{array}$ & $\begin{array}{l}\text { p.o., } 30 \mathrm{~g} \text { of } \\
\text { FA/kg of diet }\end{array}$ & $\begin{array}{l}\text { Plasma glucose, insulin, and } \\
\text { urinary glucose significantly } \\
\text { decreased }(\mathrm{P}<0.05)\end{array}$ & $\begin{array}{l}\text { (Yuan et al. } \\
\text { 1998a) }\end{array}$ \\
\hline A. auricula-judae & $\begin{array}{l}\text { Dried fruit body } \rightarrow 70 \% \text { EtOH extract } \\
\left(100^{\circ} \mathrm{C} / 6 \mathrm{~h}\right) \rightarrow \text { supernatant } \rightarrow \text { concentrated } \rightarrow \\
\text { AAE }^{* *}\end{array}$ & $\begin{array}{l}\text { Hypolipidemic activity on male } \\
\text { C57BL/6 mice in vivo }\end{array}$ & $\begin{array}{l}0.1 \%, 0.3 \%, 1 \% \\
(\mathrm{w} / \mathrm{w}) \times 8 \text { weeks } \\
(\text { r.s.: - })\end{array}$ & $\begin{array}{l}\text { Body weight, weight of adipose } \\
\text { tissue and weights of different } \\
\text { organs not significantly }(\mathrm{P}>0.05) \\
\text { different from control group }\end{array}$ & $\begin{array}{l}\text { (Reza et al. } \\
\text { 2015) }\end{array}$ \\
\hline A. polytricha* & $\begin{array}{l}\text { Fruit body } \rightarrow 95 \% \text { EtOH extract }\left(75^{\circ} \mathrm{C} / 5 \mathrm{~h}\right) \\
\rightarrow \text { residue } \rightarrow \text { hot water extraction }(3 \text { times } / 6 \mathrm{~h} \\
\text { for each }) \rightarrow \text { supernatant } \rightarrow \text { precipitated with } \\
\text { EtOH } \rightarrow \text { polysaccharide: APPs } * *\end{array}$ & $\begin{array}{l}\text { Anti-cancer activity: (1) Lung } \\
\text { cancer cells A549 (in vitro) (2) } \\
\text { subcutaneous injected A549 } \\
\text { cells BALB/c-nu mice (in vivo) }\end{array}$ & $\begin{array}{l}\text { (1) } 5-200 \mu g / \mathrm{ml} \\
\text { (2) i.p., } 1-3 \\
\mathrm{mg} / \mathrm{kg} \times 15 \text { days } \\
\text { (r.s.: normal } \\
\text { saline) }\end{array}$ & $\begin{array}{l}\text { IC50: (1) } 28.07 \mu \mathrm{g} / \mathrm{ml}(2) 0.38 \\
\mathrm{mg} / \mathrm{ml}\end{array}$ & $\begin{array}{l}\text { (Yu et al. } \\
\text { 2014) }\end{array}$ \\
\hline A. polytricha & $\begin{array}{l}\text { Fruit body } \rightarrow \text { hot water }\left(95^{\circ} \mathrm{C} / 3 \text { times } / 4 \mathrm{~h}\right) \\
\text { extract } \rightarrow \text { precipitated with } \mathrm{EtOH} \rightarrow \\
\text { polysaccharide: SPAP** }\end{array}$ & $\begin{array}{l}\text { Anti-hypercholesterolemic } \\
\text { activity on cholesterol enriched } \\
\text { diet fed Sprague-Dawley male } \\
\text { mice }\end{array}$ & $\begin{array}{l}\text { p.o., } 4.5 \mathrm{mg} / \mathrm{kg} \text { of } \\
\text { body weight }\end{array}$ & $\begin{array}{l}\text { Total cholesterol (34.6 } \pm 7.6 \%) \text {, } \\
\text { triglycerides and LDL-cholesterol } \\
\text { in blood serum significantly }(\mathrm{P}< \\
0.05 \text { ) decreased }\end{array}$ & $\begin{array}{l}\text { (Zhao et al. } \\
\text { 2015) }\end{array}$ \\
\hline A. polytricha & $\begin{array}{l}\text { Dried fruit body } \rightarrow 95 \% \mathrm{MeOH} \text { extract }(- \\
{ }^{\circ} \mathrm{C} / 7 \text { days) }\end{array}$ & $\begin{array}{l}\text { Anti-microbial activity in vitro } \\
\text { on Bacillus cereus, Escherichia } \\
\text { coli, Proteus vulgaris, } \\
\text { Staphylococcus aureus }\end{array}$ & $\begin{array}{l}20 \mathrm{mg} / \mathrm{ml}(\mathrm{r} . \mathrm{s} .:- \\
\text { ) }\end{array}$ & $\begin{array}{l}\text { Minimum inhibitory } \\
\text { concentrations (mg/ml): Bacillus } \\
\text { cereus = 3.75; Escherichia coli = } \\
\text { 3.0; Proteus vulgaris = 5.5; } \\
\text { Staphylococcus aureus = } 7.0\end{array}$ & $\begin{array}{l}\text { (Gbolagade } \\
\text { \& Fasidi } \\
\text { 2005) }\end{array}$ \\
\hline
\end{tabular}


Table 5 Continued.

\begin{tabular}{|c|c|c|c|c|c|}
\hline $\begin{array}{l}\text { Auricularia } \\
\text { species }\end{array}$ & Extraction & $\begin{array}{l}\text { Pharmacological activities / } \\
\text { Tested living system }\end{array}$ & $\begin{array}{l}\text { Routes of } \\
\text { administration/ } \\
\text { Dose }\end{array}$ & Inhibition / Decreasing ratio & References \\
\hline A. polytricha & $\begin{array}{l}\text { Dried fruit body } \rightarrow \text { hot } 95 \% \text { EtOH extract } \\
\left(75^{\circ} \mathrm{C} / 3 \text { times } / 3 \mathrm{~h}\right) \rightarrow \text { residue } \rightarrow \text { hot water } \\
\text { extract }\left(75^{\circ} \mathrm{C} / 3 \text { times } / 3 \mathrm{~h}\right) \rightarrow \text { precipitated with } \\
95 \% \text { EtOH } \rightarrow \text { APPsA-1**, APPsB-1**, } \\
\text { APPsB-2**, APPsC- } 1^{* *}\end{array}$ & $\begin{array}{l}\text { Antioxidant activity in vitro (1) } \\
\mathrm{OH}^{-}(2) \mathrm{Fe}^{3+} \text { radical } \\
\text { scavenging activity }\end{array}$ & $\begin{array}{l}4 \mathrm{mg} / \mathrm{ml} \text { (r.s.: } \\
\text { ascorbic acid, } \\
\text { ethylenediaminete } \\
\text { traacetic acid) }\end{array}$ & $\begin{array}{l}\text { Scavenging rate APPsC-1, APPsB- } \\
2 \text {, APPsB 1: }(1) \approx 70-90 \%(2) \approx 60- \\
80 \% \text {. APPsA-1: }(1) \approx 20-30 \%(2) \\
\approx 20-30 \%\end{array}$ & $\begin{array}{l}\text { (Sun et al. } \\
\text { 2010) }\end{array}$ \\
\hline A. polytricha & $\begin{array}{l}\text { Dried fruit body } \rightarrow \text { hot water extract } \\
\left(100^{\circ} \mathrm{C} / 2 \mathrm{~h}\right) \rightarrow \text { centrifuged } \rightarrow \text { supernatant } \rightarrow \\
\text { precipitated with EtOH } \rightarrow \text { centrifuged } \rightarrow \\
\text { residual in water } \rightarrow \text { dialyzed } \rightarrow \text { precipitated } \\
\text { with EtOH } \rightarrow \text { purified polysaccharides: } \\
\text { APPIIA** }\end{array}$ & $\begin{array}{l}\text { Anti-tumor activity on Sarcoma } \\
180 \text { cells implanted BALB/c } \\
\text { albino mice }\end{array}$ & $\begin{array}{l}\text { i.p., } 5 \mathrm{mg} / \mathrm{kg} \times 15 \\
\text { days }\end{array}$ & $\begin{array}{l}\text { Average tumor growth inhibition } \\
53.6 \%(\mathrm{P}<0.01)\end{array}$ & $\begin{array}{l}\text { (Yu et al. } \\
\text { 2009) }\end{array}$ \\
\hline $\begin{array}{l}\text { Auricularia sp.* } \\
\text { (Yū ěr) }\end{array}$ & $\begin{array}{l}\text { Dried fruit body } \rightarrow \text { hot } \mathrm{MeOH} \text { extract }+ \\
\text { residue extracted with hot } 70 \% \mathrm{EtOH}+ \\
\text { residue extracted with hot water }(4 \text { times/5h) } \\
\rightarrow \text { purified, precipitated with EtOH } \rightarrow \text { U-3- } \\
\text { EP** } \rightarrow \text { fractionated by CTAB } \rightarrow \text { U-3-A** }\end{array}$ & $\begin{array}{l}\text { Anti-inflammatory activity on } \\
\text { Sprague Dawley mice (1) } \\
\text { Carrageenin induced edema (2) } \\
\text { Scald induced hyperalgesia }\end{array}$ & $\begin{array}{l}\text { i.p., } 25 \mathrm{mg} / \mathrm{kg} \\
\text { (r.s.: } \\
\text { phenylbutazone) }\end{array}$ & $\begin{array}{l}\text { (1) Hind paw thickness } 50.1 \%(2) \\
\text { Pain threshold } 64.1 \%\end{array}$ & $\begin{array}{l}\text { (Kiho et al. } \\
\text { 1985) }\end{array}$ \\
\hline $\begin{array}{l}\text { Auricularia sp.* } \\
\text { (Yū ěr) }\end{array}$ & $\begin{array}{l}\text { Dried fruit body } \rightarrow \text { hot } \mathrm{MeOH} \text { extract }+ \\
\text { residue extracted with hot } 70 \% \mathrm{EtOH}+ \\
\text { residue extracted with hot water }(4 \text { times/5h) } \\
\rightarrow \text { purified, precipitated with EtOH } \rightarrow \text { U-3- } \\
\text { EP** } \rightarrow \text { fractionated by CTAB } \rightarrow \text { U-3-A** }\end{array}$ & $\begin{array}{l}\text { Anti-tumor activity on Sarcoma } \\
180 \text { cells implanted ddY male } \\
\text { mice }\end{array}$ & $\begin{array}{l}\text { i.p., } 25 \mathrm{mg} \times 10 \\
\text { days }\end{array}$ & Average tumor weight $86 \%$ & $\begin{array}{l}\text { (Ukai et al. } \\
\text { 1983b) }\end{array}$ \\
\hline A. thailandica & $\begin{array}{l}\text { Dried fruit body } \rightarrow 100 \% \mathrm{MeOH} \text { extract } \\
\left(45^{\circ} \mathrm{C} / 40 \text { min. }\right)\end{array}$ & $\begin{array}{l}\text { Antioxidant activity in vitro (1) } \\
\text { ABTS (2) DPPH radical } \\
\text { scavenging activity }\end{array}$ & $\begin{array}{l}1-5 \mathrm{mg} / \mathrm{ml}(\mathrm{r} . \mathrm{s} .: \\
\text { ascorbic acid }\end{array}$ & $\begin{array}{l}\text { EC50 (1) } 835 \mu \mathrm{g} / \mathrm{ml}(2) 342 \\
\mu \mathrm{g} / \mathrm{ml}\end{array}$ & $\begin{array}{l}\text { (Bandara et } \\
\text { al. 2017a) }\end{array}$ \\
\hline
\end{tabular}

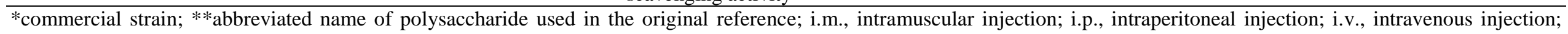
m.w., molecular weight; r.s., reference standard; r.t., room temperature; p.o., oral administration; s.c., subcutaneous injection.

Polysaccharides consisting of more than one sugar or sugar derivative are called heteropolysaccharides (Enshasy \& Hatti-Kaul 2013, Davidson 2018). Heteropolysaccharides composed of $\alpha-(1 \rightarrow 3)$-linked D-mannopyranosyl backbones (Fig. 3.1), with the branches of D-glucopyranosuronic acid, D-mannopyranose and D-xylopyranose residues [i.e. $\alpha$ - $(1 \rightarrow 3)$-glucuronoxylomannans], which contained a small proportion of D-glucopyranose, were reported from A. auricula-judae (Sone et al. 1978, Misaki et al. 1981, Ukai et al. 1982, Misaki \& Kakuta 1995). Though the $\alpha$-(1 $\rightarrow 3$ )-linked Dmannopyranosyl backbone was reported for several fungal heteropolysaccharides (Sone et al. 1978), $\alpha$-(1 $\rightarrow 3$ )-glucuronoxylomannans were found to be specific to jelly mushrooms (Kakuta et al. 1979, Wasser 2002). $\alpha$-Configuration of the $(1 \rightarrow 3)$-linked D-mannopyranosyl residues possess a single chain, which leads to the absence of its anti-tumor potential, while the $\beta$-configuration, which has a triple helical structure, is necessary to produce 
anti-tumor and immunomodulating activity (Misaki et al. 1981, Misaki \& Kakuta 1995, Enshasy \& Hatti-Kaul 2013). Heteropolysaccharides with $\beta$-configuration glucopyranosyl residues were also detected from A. auricula-judae (Table 4) (Ma et al. 2008, Yang et al. 2011). The water-soluble $\beta$ D-glucan isolated from $A$. auricula-judae was composed of $\beta-(1 \rightarrow 4)$-linked D-glucopyranosyl backbone with $\beta-(1 \rightarrow 6)$-linked glucopyranosyl side groups at C-6, including glucuronic acid (Ma et al. 2008). A backbone similar to $\beta$-D-glucan isolated from A. auricula-judae (Fig. 3.2) (Ma et al. 2008) was observed in the polysaccharopeptide Krestin isolated from Coriolus versicolor (Ooi \& Liu 2000). Although the branching pattern of $\beta-(1 \rightarrow 6)$-linked glucopyranosyl side groups of $\beta$-Dglucan isolated from $A$. auricula-judae and Krestin isolated from $C$. versicolor differed from one another, both polysaccharides acted as potential anti-tumor agents (Ma et al. 2010, Maehara et al. 2012).

The majority of polysaccharides isolated from fungi are water insoluble; among water-soluble polysaccharides, most are heteropolysaccharides, having a complicated structure and thus making it difficult to elucidate their primary and secondary structures (Ma et al. 2008). In general, mushroom polysaccharides are highly diversified in their sugar composition, main chain polymer structure, degree of branching, conformation, molecular weight, and other physical properties, all of which significantly influence the bioactivity and mode of action of the polysaccharide (Enshasy \& HattiKaul 2013). It should be noted that different extraction methods and different materials affect the polysaccharide concentration of $A$. auricula ( $\mathrm{Li}$ et al. 2019). The results showed that the enzymebased extraction was better than either ultrasonic-assisted extraction or water extraction technique. The extraction process of polysaccharides affects the bioactive molecule content of the mushroom extracts and then their biological activities, for any therapeutic target (Li et al. 2019).

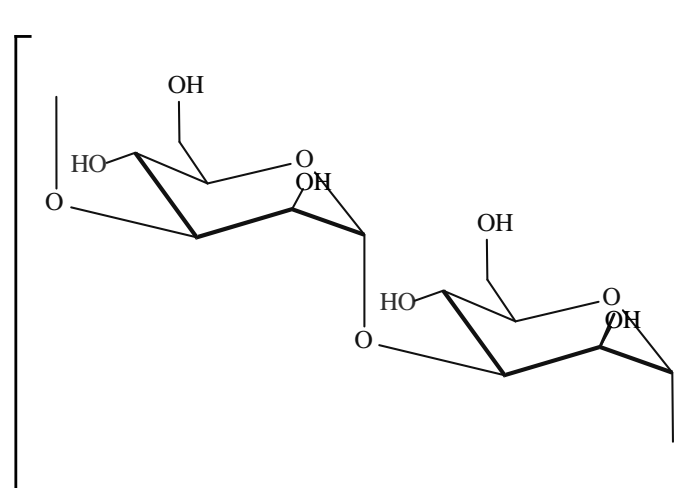

(1) $\alpha$-(1 $\rightarrow 3)$-linked-D-mannopyranosyl backbone
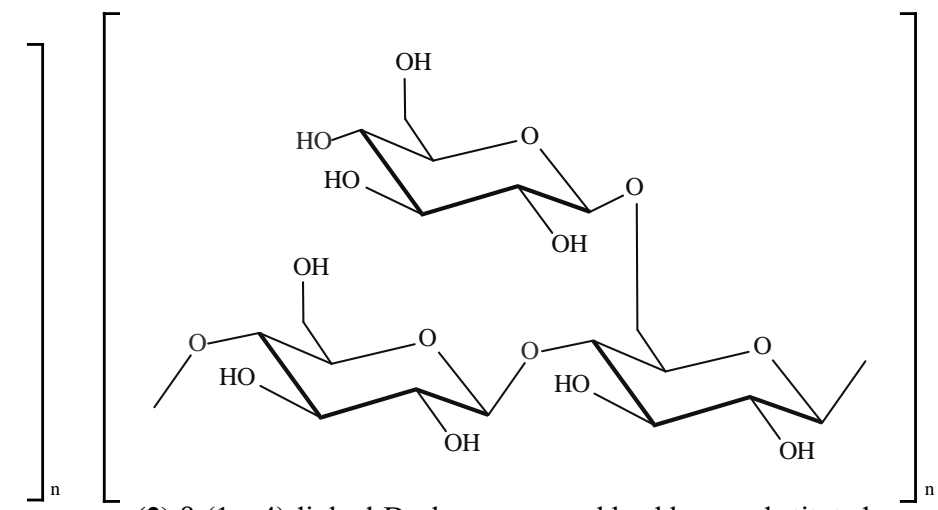

(2) $\beta$-(1 $\rightarrow 4)$-linked-D-glucopyranosyl backbone substituted $\beta-(1 \rightarrow 6)$-linked glucopyranosyl side chanis at C-6 (AAG)

Figure 3 - Chemical structures of various types of backbones of heteropolysaccharides from Auricularia auricula-judae. $1 \alpha-(1 \rightarrow 3)$-linked D-mannopyranosyl backbone (Sone et al. 1978, Misaki et al. 1981, Ukai et al. 1982, 1983a, b). 2 AAG (Ma et al. 2008, 2010)

\section{Bioactive polysaccharide properties of Auricularia and potential novel drug therapeutic uses}

The following section describes the different bioactive properties of polysaccharides derived from Auricularia species, including anti-cancer, anti-tumor, immunomodulatory, anti-bacterial, anti-viral, antioxidant, hypoglycemic, and anti-hypercholesterolemic properties. It should be noted that it is currently unknown whether all Auricularia species possess each of these properties. In some cases, the polysaccharides have to be chemically modified (e.g. sulfation) to enhance a certain property, such as the anti-viral properties of A. auricula polysaccharides (Nguyen et al. 2012a). Although certain of these compounds are already used in treating disease, there are others that are still in experimental stages of development, determining potential medicinal and pharmaceutical applications in the future. In addition to the different medical properties of these polysaccharides, 
the factors that may influence their activity are also discussed here. The properties of Auricularia polysaccharides are summarized in Fig. 4.

\section{Polysaccharides with anti-cancer or anti-tumor properties}

Many Auricularia polysaccharides have anti-tumor properties that are mediated through $(1 \rightarrow 3)-\beta$-D-glucan molecules, which induce cancer cell apoptosis [reviewed in (Meng et al. 2016)]. Auricularia polytricha polysaccharides were shown to inhibit cancer activities in A549 human lung cancer cells by inhibiting DNA synthesis, cell cycle arrest, and cell proliferation, thus indicating their potential as a chemotherapeutic agent in combating lung cancer ( $\mathrm{Yu}$ et al. 2014). The incorporation of these compounds into unique drug delivery systems can enhance their effectiveness. One such example is the use of the Huaier granules for gastrointestinal cancer (Ma et al. 2018). The folic acid conjugated Auricularia auricula polysaccharide, cisdiaminedichloroplatinum complex, is a good example of an enhanced polysaccharide that utilizes a drug delivery system to increase its anti-tumor potency while reducing its cytotoxicity in treating cervical carcinoma (Qiu et al. 2018). Additionally, water, ethanol, ethyl acetate, dichloromethane, and butanol can be used as solvents for the extraction of bioactive compounds from Auricularia, i.e., $(1 \rightarrow 3)$ - and $(1 \rightarrow 6)-\beta-D$-glucan-containing polysaccharides are extracted from $A$. auriculajudae in dichloromethane fraction, which has been identified as an anti-tumor candidate for treating sarcoma 180 cells (Reza et al. 2011). Auricularia polytricha polysaccharides have also been shown to have anti-tumor effects against sarcoma (Yu et al. 2009). An in vivo anti-tumor test conducted in mice with sarcoma 180 tumors showed that the polysaccharides have anti-tumor effects by activating the functions of macrophages (Yu et al. 2009). A polysaccharide main chain composed of $(1 \rightarrow 4)$-linked D-glucopyranosyl and $(1 \rightarrow 6)$-linked D-glucopyranosyl with a branch at O-6 from A. auricula-judae was also shown to have anti-tumor capabilities which are mediated through cancer cell apoptosis [reviewed in (Meng et al. 2016)]. The cytotoxic properties of A. auriculajudae are also used to inhibit cancer cells derived from the HCT116 cell line, while depending on the extraction method that renders polarity of the bioactive compounds provided them with additional cytotoxic [non polar (n-hexane) extract], anti-oxidative [ethyl acetate and methanol extracts], and anti-diabetics [ethyl acetate extract] properties (Elkhateeb et al. 2018).

\section{Polysaccharides with anti-bacterial properties}

Hot water extraction, followed by alcohol precipitation, was used to collect crude polysaccharides of $A$. auricula-judae, which were then investigated for anti-microbial capacities (Cai et al. 2015). These polysaccharides derived from A. auricula-judae were shown to inhibit the activities of Escherichia coli and Staphylococcus aureus, confirming their anti-microbial activity (Cai et al. 2015). Chitosan is an organic polymer and a linear polysaccharide [ $\beta-(1 \rightarrow 4)$-linked Dglucosamine and $\mathrm{N}$-acetyl-D-glucosamine] that is commercially available and used to treat many diseases, including obesity and hypercholesterolemia. However, chitosan exhibiting a higher degree of deacetylation compared to commercially available forms was extracted from Auricularia species in a recent study (Chang et al. 2019). The work of Chang et al. (2019) showed that the high deacetylation power of Auricularia chitosan inhibited the growth of E. coli and S. aureus, further confirming the anti-bacterial properties of Auricularia polysaccharides. Moreover, adding low molecular weight chitosan (positively charged) to A. auricula polysaccharides (negatively charged) to create novel polyelectrolyte complex nanoparticles proved to be an ideal delivery system for protein drugs, expanding the potential uses of Auricularia polysaccharides in developing novel drug therapies (Xiong et al. 2016).

\section{Polysaccharides with anti-viral properties}

Auricularia auricula has been used to extract total A. auricula polysaccharides (known as AAPt), which can then be sulfated to enhance their anti-viral activity. The ability of sulfated AAPt in inhibiting the activity of Newcastle Disease Virus on cultured chicken embryo fibroblasts measured using MTT [3-(4,5-Dimethylthiazol-2-yl)-2,5-Diphenyltetrazolium Bromide] assay 
provides evidence for its anti-viral activity (Nguyen et al. 2012a). The authors refer to the sulfated AAPt as "the component of a new-type anti-viral drug" (Nguyen et al. 2012a). Studies on anti-viral properties of Auricularia species are still rare. However, the proven anti-viral properties of many fungal species within Basidiomycota in inhibiting a wide range of viruses including BoHV-1, H1N12, HBV, HCV, HIV, Influenza, Polio, and Vaccinia highlight the potential for Auricularia species to provide similar defenses against these viruses, and their potential applications in treating a wide range of viral diseases (Linnakoski et al. 2018).

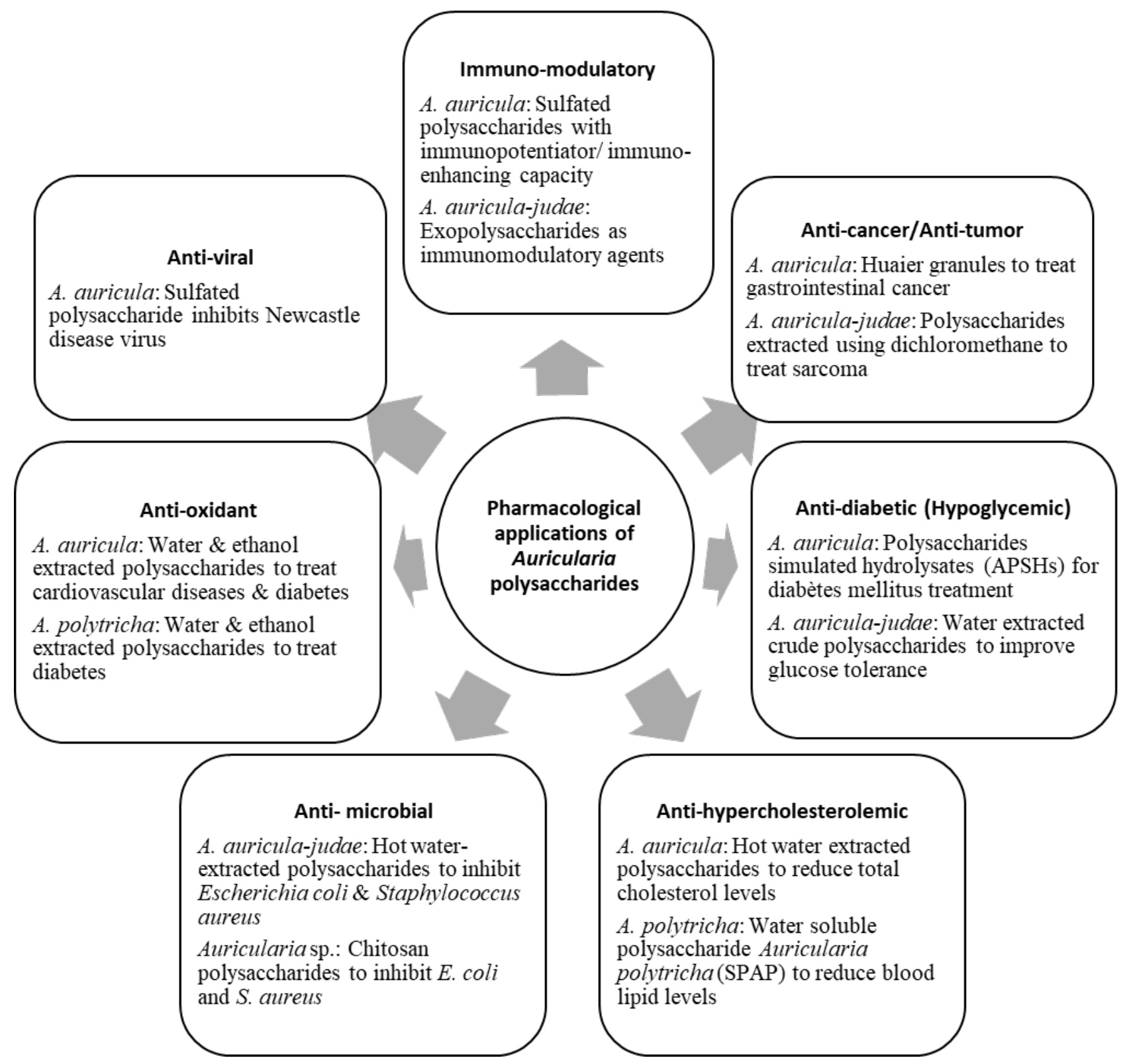

Figure 4 - Bioactive properties of the polysaccharides extracted from different Auricularia species. The figure summarizes anti-cancer / anti-tumor (Reza et al. 2011, Ma et al. 2018), anti-diabetic (Yuan et al. 1998b, Lu et al. 2018b), anti-hypercholesterolemic (Chen et al. 2008, Zhao et al. 2015), anti-microbial (Cai et al. 2015, Xiong et al. 2016, Chang et al. 2019), antioxidant (Wu et al. 2010, Avci et al. 2016, Hu et al. 2017), anti-viral (Nguyen et al. 2012a) and immunomodulatory (Nguyen et al. 2012b, Zhang et al. 2018a) properties of polysaccharides from Auricularia species.

\section{Polysaccharides with immunomodulatory properties}

The AAPt from A. auricula also showcase immuno-enhancing activities when modified by sulfation using the chlorosulfonic acid-pyridine method (Nguyen et al. 2012b). Both in vitro and in 
vivo experiments suggest the use of sulfated AAPt (sAAPt) as an "immunopotentiator" (Nguyen et al. 2012b). A submerged culture of A. auricula-judae containing exopolysaccharides (CEPSN-1 and CEPSN-2) with $(1 \rightarrow 4)$ - $\alpha$-D-glucose backbones was used by Zhang et al. (2018a) to demonstrate their immunomodulatory activities. Even though the polysaccharide backbone structures differed between the fruiting bodies and the submerged cultures of A. auricula-judae, the exopolysaccharides showed potential immunomodulatory activities (Zhang et al. 2018a). This observation suggests that immunomodulatory drugs could be generated using exopolysaccharides from submerged cultures of A. auricula-judae.

Inflammation is a natural response to a weakened immune system, and many Auricularia species such as $A$. polytricha have anti-inflammatory properties that arise not only from their polysaccharides but also other bioactive compounds such as carotenoids, fatty acids, vitamins, and phenolic compounds (Muszynska et al. 2018). For example, A. auricular-judae polysaccharides have shown protective effects in rats who have acute lung injury induced by lipopolysaccharide which was illustrated by the reduced lung edema and lowered levels of inflammatory mediators (Li et al. 2015). Similarly, A. auricular-judae polysaccharides were able to inhibit edema (carrageenininduced) and hyperalgesia (scald-induced) in rats through its anti-inflammatory properties (Ukai et al. 1983a). The medicinal implications of Auricularia polysaccharides were further illustrated using a rat model of non-alcoholic fatty liver disease who were treated with a diet containing water extract of $A$. polytricha that is rich in polysaccharides, phenolic compounds and tannins with antiinflammatory properties. The rats who received the A. polytricha-containing diet showed slowed progression of liver disease in association with elevated anti-oxidative markers suggesting the use of Auricularia supplementation as a potential remedy (Chiu et al. 2014). As the same disease model showed attenuation of lipid deposition, the study also provides implications of Auricularia supplementation in treating obesity. The anti-inflammatory as well as antioxidant (discussed below) properties of Auricularia polysaccharides may also render cardio-protective properties to them providing insights into using these polysaccharides for cardiovascular diseases (Hao 2014).

\section{Polysaccharides with antioxidant properties}

Auricularia species have been reported to possess polysaccharides with antioxidant properties, and examples include A. auricula (Xu et al. 2016) and A. polytricha (Avci et al. 2016). In addition to different species providing different antioxidants, the potential for antioxidant production can also vary according to the strain of a given Auricularia species (Xu et al. 2016). Regardless of the differences observed among varieties, the proven antioxidant ability of $A$. auricula polysaccharides in improving heart functions in a mouse model (Wu et al. 2010) have significant implications for the use of Auricularia polysaccharides as pharmaceutical antioxidants for cardiovascular disease treatments. In addition, experimental studies using diabetic mice (in vivo) showed that the polysaccharides of $A$. auricula were able to modulate anti-oxidative systems and treat diabetic conditions ( $\mathrm{Hu}$ et al. 2017). Polysaccharides from A. polytricha were extracted using ethanol and distilled water, and an assessment of their antioxidant activity demonstrated that water-based extraction was more efficient for obtaining higher antioxidant activity when compared to ethanol extraction (Avci et al. 2016). These animal model-based studies as well as in vitro assays provide further evidence that polysaccharides of Auricularia may be used in humans after their advancement into clinical trials. Antioxidants may perform its functions through radical scavenging. For example, sulfated neutral and acidic A. auricula polysaccharides showed high radical scavenging abilities which have tremendous implications in disease conditions caused by oxidative stress and generation of free radicals such as Alzheimer's disease and cancer (Zhang et al. 2011). The free radical scavenging properties of $A$. auricula were shown to be useful not only as a medicine but also a functional food when it used as a pickled product (Khaskheli et al. 2015).

\section{Polysaccharides with anti-hypercholesterolemic properties}

Polysaccharides extracted from Auricularia have the potential to reduce cholesterol, thus 
indicating the potential for use in the treatment of hypercholesterolemia. Several animals, including rats and mice, have been used in experiments demonstrating the anti-hypercholesterolemic properties of Auricularia polysaccharides. For example, a diet-induced hypercholesterolemic rat was orally administered with soluble polysaccharides from Auricularia polytricha, resulting in a reduction in blood lipid levels, thereby confirming the natural anti-hypercholesterolemic properties of Auricularia polytricha (Table 5) (Zhao et al. 2015). Similarly, hypercholesterolemic rats fed with an A. auricula diet in dry powder form had reduced levels of serum LDL cholesterol, as well as lower fecal neutral steroids and bile acids, all of which suggest its effectiveness in reducing hypercholesterolemic activity (Cheung 1996). Further evidence for the hypocholesterolemic capacities of Auricularia was provided when hot water-extracted polysaccharides from A. auricula were orally administered to hypercholesterolemic mice, and total cholesterol and high-density lipoprotein cholesterol were significantly reduced (Chen et al. 2008).

\section{Polysaccharides with hypoglycemic properties}

Bio-compounds with hypoglycemic properties are important in the treatment of diabetes. Auricularia auricula polysaccharides-simulated-hydrolysates are bio-compounds with hypoglycemic properties, and intragastric administration of A. auricula polysaccharides-simulatedhydrolysates has been suggested as a potential treatment for diabetes mellitus ( $\mathrm{Lu}$ et al. 2018b). A model incorporating diabetic mice (diet-streptozotocin-induced) was used to test the anti-diabetic capabilities of $A$. auricula polysaccharides. These polysaccharides were reported to reduce blood glucose levels in the mice, producing a result similar to the well-known anti-diabetic medicine Metformin (Hu et al. 2017); however, further studies are required to verify these results. Moreover, polysaccharides composed of glucose, 2-deoxy-glucose, arabinose, xylose, mannose, and glucosamine from A. auricula were shown to effectively treat rats with type 2 diabetes mellitus by inhibiting the oxidative stress response signaling pathway ( $\mathrm{Lu}$ et al. 2018a), a pathological mechanism associated with diabetes (Tiwari et al. 2013).

Research conducted previously provides evidence that the extraction method of Auricularia polysaccharides is critical to their anti-diabetic properties and glucose tolerance (Yuan et al. 1998b). According to the study, in contrast to the anti-diabetic properties of crude polysaccharides and neutral polysaccharide fractions extracted from A. auricula-judae, the acidic polysaccharide fraction derived from $A$. auricula-judae had no impact on diabetes when tested on mice. The administration of crude polysaccharides but not neutral polysaccharides improved glucose tolerance (Yuan et al. 1998b). In addition to maintaining blood glucose levels, Auricularia has also been associated with the treatment of nerve damage linked to diabetes. Aldose Reductase Inhibitors are a drug used for the treatment of this type of nerve damage and A. auricula-judae polysaccharide extracts exhibited similar actions to Aldose Reductase inhibitory activity, suggesting its application as an anti-diabetic drug (Wu \& Xu 2015).

\section{Additional bioactive compounds and their functions}

\section{Proteins}

After polysaccharides, proteins are the most abundant bio-compounds in Auricularia species representing its proximate composition. Proximate composition analysis of A. auricula-judae has shown that it contains $8.36 \%$ of proteins and $33.04 \%$ of total proteins are composed of essential amino acids (Afiukwa et al. 2015). Some of the proteins and peptide sequences isolated from Auricularia appear to have medical and pharmaceutical properties similar to polysaccharides (Sheu et al. 2004, Agyei \& Danquah 2011). The peptide sequences from Auricularia species that have pharmacological properties are called bioactive peptides (Agyei \& Danquah 2011). An example of a bioactive protein is an immunomodulatory protein extracted from the basidiocarp of A. polytricha found to have immunostimulant abilities (Sheu et al. 2004). Further experimentation has shown that the immunomodulatory proteins isolated from A. polytricha have properties of pharmaceutical and 
stable immune stimulants such as tolerance to acids and alkali, heat and freezing, and dehydration (Chang et al. 2007).

\section{Exo-biopolymers}

Exo-biopolymers, also known as exopolymers, are biopolymers generated by an organism, such as a fungus, and are secreted into the environment. In particular, Auricularia species are known to produce a wide range of biopolymers (Yang et al. 2002, Xu \& Yun 2003, Jeong et al. 2007). These compounds have been shown to have potential in the treatment of hyperlipidemia, in a study carried out on hyperlipidemic rats (Yang et al. 2002). In these rats, an exo-biopolymer, isolated from A. polytricha and composed of carbohydrates (77.5\%) and proteins (22.5\%), reduced plasma LDL cholesterol by 70\% in rats (Yang et al. 2002).

\section{Melanin}

Apart from the major edible and medicinal components of Auricularia species, melanin is one compound that has applications in the food industry, as well as the pharmacological, medicinal, and cosmetic fields, with potential for novel claims within these industries (Hyde et al. 2010, Sun et al. 2016). Submerged cultures of $A$. auricula with glucose, tyrosine, peptone, and $\mathrm{CaCO}_{3}$ additives is a reliable method for the large-scale production of natural melanin (Zhang et al. 2015b). Sun et al. (2016) demonstrated that the use of the yeast extracts tyrosine and lactose during the microbial submerged fermentation process of the melanin extraction and purification protocol could significantly increase the amount of purified melanin by $A$. auricula. The capacity of $A$. auricula submerged cultures (with tyrosine additives) to produce melanin pigments has also been demonstrated (Wu et al. 2018, Zou \& Ma 2018). Thus, A. auricula also has applications in the food industry as a colorant (Zou \& Ma 2018). Additionally, a mix of methanol (1\%), peanut oil (0.25\%), and stearic acid $(1.0 \%)$ or palmitic acid $(0.5 \%)$ has been shown to increase the biosynthesis of melanin 1.53 times that of submerged cultures of $A$. auricula (Wu et al. 2018). Melanin isolated from A. auricula also possesses anti-microbial or anti-biofilm properties, as illustrated by the inhibition of biofilms formed by E. coli K-12, Pseudomonas aeruginosa PAO1, and Pseudomonas fluorescens P-3 (Bin et al. 2012).

\section{Adenosine}

Adenosine is a nucleoside which is a major constituent of DNA and RNA. Adenosine from A. auricula extracted using water, methanol, or butanol showed a potent inhibitory effect for rabbit platelet aggregation and suggests anti-platelet functions (Murata et al. 2004). Auricularia auricula and $A$. polytricha with adenosine contents of 133 and $154 \mu \mathrm{g} / \mathrm{g}$ of dry fungus, respectively, have been shown to inhibit ADP-induced aggregation of platelets both on a rat model and in human platelets, thereby indicating their potential for application in humans (Agarwal et al. 1982). These anti-platelet or anti-aggregant properties are of great interest for their capacity to inhibit thrombus formation, and thus can be used as a potential drug therapy for cerebrovascular or cardiovascular diseases.

\section{Polyphenolic compounds}

Ethanol extract of A. auricula containing polyphenolic compounds [16\% (g/g dry weight) without polysaccharides, water-soluble fiber, and proteins] was found to decrease total cholesterol levels and atherosclerosis indexes in mice, indicating the hypocholesterolemic effects of these polyphenolic compounds (Chen et al. 2011). According to the authors, the study provides evidence for the application of A. auricula not only to prevent but also to treat cardiovascular disease.

\section{Conclusion and Future Perspectives}

Among the bioactive chemicals of Auricularia species, polysaccharides are the most promising, possessing anti-cancer, anti-bacterial, anti-viral, immunomodulatory, antioxidant, hypoglycemic, and anti-hypercholesterolemic properties. In addition, exo-biopolymers, melanin, 
polyphenolic compounds, and proteins have also been recognized as potential pharmacological agents that can be further developed into novel drugs for treating human disease. However, proteins have been shown to be of great potential as a source of novel compounds and treatments, and thus should be more extensively researched in the future. The bioactive compounds of Auricularia species hold promise for the development of future drug therapies and supplements for many human diseases. However, much of the research regarding these treatments has been conducted on animal models and thus requires further validation using clinical trials and human patients.

\section{Acknowledgements}

Peter E. Mortimer would like to thank the National Science Foundation of China (NSFC) for partially funding this research under the project codes 41761144055 and 41771063 , as well as the South East Asian Biodiversity Research Institute for financial support (grant no. Y4ZK111B01). K.D. Hyde would like to thank the Thailand Research Fund for the grant "Domestication and bioactive evaluation of Thai Hymenopellis, Oudemansiella, Xerula and Volvariella species (basidiomycetes)" Grant No.: DBG6180033 for funding this work.

\section{References}

Afiukwa C, Ebem E, Igwe D. 2015 - Characterization of the proximate and amino acid composition of edible wild mushroom species in Abakaliki, Nigeria. American Association for Science and Technology Journal of Bioscience 1, 20-25.

Afiukwa C, Oko A, Afiukwa J, Ugwu O et al. 2013 - Proximate and mineral element compositions of five edible wild grown mushroom species in Abakiliki, South East Nigeria. Research Journal of Pharmaceutical Biological and Chemical Sciences 4, 1056-1064.

Agarwal KC, Russo FX, Parks Jr RE. 1982 - Inhibition of human and rat platelet aggregation by extracts of Mo-er (Auricularia auricula). Thrombosis and Haemostasis 47, 162-165.

Agyei D, Danquah MK. 2011 - Industrial-scale manufacturing of pharmaceutical-grade bioactive peptides. Biotechnology Advances 29, 272-277.

Aletor VA. 1995 - Compositional studies on edible tropical species of mushrooms. Food Chemistry 54, 265-268.

Alexander H. 2013 - Morphology and Classification. In: Chang S T, Hayes W A (eds) The biology and cultivation of edible mushrooms. Academic Press, London, UK, pp 3-33.

Avci E, Çağatay G, Avci GA, Suiçmez M et al. 2016 - An edible mushroom with medicinal significance; Auricularia polytricha. Hittite Journal of Science and Engineering 3, 111-116.

Bandara AR, Karunarathna SC, Mortimer PE, Hyde KD et al. 2017a - First successful domestication and determination of nutritional and antioxidant properties of the red ear mushroom Auricularia thailandica (Auriculariales, Basidiomycota). Mycological Progress 16, 1029-1039.

Bandara AR, Karunarathna SC, Phillips AJ, Mortimer PE et al. 2017b - Diversity of Auricularia (Auriculariaceae, Auriculariales) in Thailand. Phytotaxa 292, 19-34.

Bin L, Wei L, Xiaohong C, Mei J et al. 2012 - In vitro antibiofilm activity of the melanin from Auricularia auricula, an edible jelly mushroom. Annals of Microbiology 62, 1523-1530.

Cai M, Lin Y, Luo YL, Liang HH et al. 2015 - Extraction, antimicrobial, and antioxidant activities of crude polysaccharides from the wood ear medicinal mushroom Auricularia auricula-judae (higher Basidiomycetes). International Journal of Medicinal Mushrooms 17, 591-600.

Carrasco J, Zied DC, Pardo JE, Preston GM et al. 2018 - Supplementation in mushroom crops and its impact on yield and quality. AMB Express 8, 146.

Chang AKT, Frias RR, Alvarez LV, Bigol UG et al. 2019 - Comparative antibacterial activity of commercial chitosan and chitosan extracted from Auricularia sp. Biocatalysis and Agricultural Biotechnology 17, 189-195. 
Chang HH, Chien PJ, Tong MH, Sheu F. 2007 - Mushroom immunomodulatory proteins possess potential thermal/freezing resistance, acid/alkali tolerance and dehydration stability. Food Chemistry 105, 597-605.

Chang ST, Hayes WA. 2013 - The biology and cultivation of edible mushrooms. Academic Press, London, UK.

Chang ST, Miles PG. 2004 - Mushrooms: cultivation, nutritional value, medicinal effect, and environmental impact. $2^{\text {nd }}$ edn. CRC Press, Boca Raton, Florida.

Chang ST, Wasser S. 2017 - The cultivation and environmental impact of mushrooms. Oxford Research Encyclopedia of Environmental Science, 1-37.

Chang YS, Lee SS. 2004 - Utilisation of macrofungi species in Malaysia. Fungal Diversity 15, 1522.

Chen G, Luo YC, Ji BP, Li B et al. 2008 - Effect of polysaccharide from Auricularia auricula on blood lipid metabolism and lipoprotein lipase activity of ICR mice fed a cholesterol-enriched diet. Journal of Food Science 73, H103-H108.

Chen g, Luo YC, Ji BP, Li B et al. 2011 - Hypocholesterolemic effects of Auricularia auricula ethanol extract in ICR mice fed a cholesterol-enriched diet. Journal of Food Science and Technology 48, 692-698.

Cheung PCK. 1996 - The hypocholesterolemic effect of two edible mushrooms: Auricularia auricula (tree-ear) and Tremella fuciformis (white jelly-leaf) in hypercholesterolemic rats. Nutrition Research 16, 1721-1725.

Cheung PCK. 1997 - Dietary fibre content and composition of some edible fungi determined by two methods of analysis. Journal of the Science of Food and Agriculture 73, 255-260.

Cheung PCK. 2013 - Mini-review on edible mushrooms as source of dietary fiber: Preparation and health benefits. Food Science and Human Wellness 2, 162-166.

Chinamushroombusinessnetwork. 2018 - Investigation Report on Auricularia auricula Industry in 2017. http://zixun.mushroommarket.net/201808/16/185667.html. Accessed 06.172019

Chiu WC, Yang HH, Chiang SC, Chou YX et al. 2014 - Auricularia polytricha aqueous extract supplementation decreases hepatic lipid accumulation and improves antioxidative status in animal model of nonalcoholic fatty liver. Biomedicine 4, 12.

Crisan EV, Sands A. 1978 - 6-Nutritional Value. In: Chang S T, Hayes W A (eds) The Biology and Cultivation of Edible Mushrooms. Academic Press, pp 137-168.

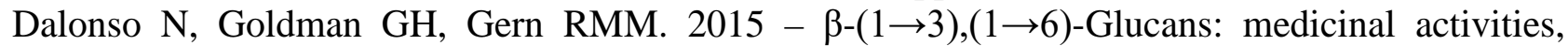
characterization, biosynthesis and new horizons. Applied Microbiology and Biotechnology 99, 7893-7906.

Damte D, Reza MA, Lee SJ, Jo WS et al. 2011 - Anti-inflammatory activity of dichloromethane extract of Auricularia auricula-judae in RAW264.7 cells. Toxicological Research 27, 11-14.

Davidson EA. $2018 \quad-\quad$ Carbohydrate. Encyclopædia Britannica, Inc. http://www.britannica.com/science/carbohydrate/Heteropolysaccharides. Accessed 09 January 2019

De Silva DD, Rapior S, Fons F, Bahkali AH et al. 2012a - Medicinal mushrooms in supportive cancer therapies: an approach to anti-cancer effects and putative mechanisms of action. Fungal Diversity 55, 1-35.

De Silva DD, Rapior S, Hyde KD, Bahkali AH. 2012b - Medicinal mushrooms in prevention and control of diabetes mellitus. Fungal Diversity 56, 1-29.

De Silva DD, Rapior S, Sudarman E, Stadler M et al. 2013 - Bioactive metabolites from macrofungi: ethnopharmacology, biological activities and chemistry. Fungal Diversity 62, 140.

Deka AC, Sarma I, Dey S, Sarma TC. 2017 - Antimicrobial properties and phytochemical screening of some wild macrofungi of Rani-Garbhanga reserve forest area of Assam, India. Advances in Applied Science Research 8, 17-22.

Du P, Cui BK, Zhang CF, Dai YC. 2013 - Genetic diversity of wild Auricularia auricula-judae revealed by ISSR analysis. Biochemical Systematics and Ecology 48, 199-205. 
Duc PH. 2005 - Mushrooms and cultivation of mushrooms in Vietnam. Mushroom Growers' Handbook 2 Shiitake Cultivation, MushWorld, Republic of Korea, pp 1-11.

Dupont J, Dequin S, Giraud T, Le Tacon F et al. 2017 - Fungi as a Source of Food. In: The Fungal Kingdom. Microbiology Spectrum 5, FUNK-0030-2016.

Elkhateeb WA, El-Hagrassi AM, Fayad W, El-Manawaty MA et al. 2018 - Cytotoxicity and hypoglycemic effect of the Japanese Jelly mushroom Auricularia auricula-judae. Chemistry Research Journal 3, 123-133.

Enshasy HAE, Hatti-Kaul R. 2013 - Mushroom immunomodulators: unique molecules with unlimited applications. Trends in Biotechnology 31, 668-677.

Fan L, Zhang S, Yu L, Ma L. 2006 - Evaluation of antioxidant property and quality of breads containing Auricularia auricula polysaccharide flour. Food Chemistry 101, 1158-1163.

Friedman M. 2016 - Mushroom polysaccharides: Chemistry and antiobesity, antidiabetes, anticancer, and antibiotic properties in cells, rodents, and humans. Foods 5, 80.

Fuller R, Buchanan P, Roberts M. 2004 - Maori Knowledge of Fungi / Mātauranga o Ngā Harore. In: McKenzie EHC (ed) Introduction to Fungi of New Zealand. Fungi of New Zealand, vol 1. Fungal Diversity Press, pp 81-118.

Fuller R, Buchanan P, Roberts M. 2005 - Medicinal uses of fungi by New Zealand Maori people. International Journal of Medicinal Mushrooms 7, 402.

Gbolagade JS, Fasidi IO. 2005 - Antimicrobial activities of some selected Nigerian mushrooms. African Journal of Biomedical Research 8, 83-87.

Guo G, Chen Y, Tong C, Qu M et al. 2015 - A water-soluble polysaccharide from Auricularia auricula root and its hepatoprotective effect against acute carbon tetrachloride-induced liver injury in mice. Science and Technology of Food Industry 36, 341-344.

Guzmán G. 2008 - Diversity and use of traditional Mexican medicinal fungi. A review. International Journal of Medicinal Mushrooms 10, 209-217.

Hao H. 2014 - Effect effects of Auricularia auricula polysaccharides on exhaustive swimming exercise-induced oxidative stress in mice. Tropical Journal of Pharmaceutical Research 13, 1845-1851.

Hapuarachchi KK, Elkhateeb WA, Karunarathna SC, Cheng CR et al. 2018a - Current status of global Ganoderma cultivation, products, industry and market. Mycosphere, Journal of Fungal Biology 9, 1025-1052.

Hapuarachchi KK, Karunarathna S, Phengsintham P, Kakumyan P et al. 2018b - Amauroderma (Ganodermataceae, Polyporales)-bioactive compounds, beneficial properties and two new records from Laos. Asian Journal of Mycology 1, 121-136.

Hapuarachchi KK, Wen TC, Jeewon R, Wu XL et al. 2016 - Mycosphere Essays 7. Ganoderma lucidum - are the beneficial anti-cancer properties substantiated? Mycosphere, Journal of Fungal Biology 7, 305-332.

Hassan FRH, Medany GM. 2012 - Studies on submerged culture conditions for mycelial biomass production of wood ears mushroom (Auricularia polytricha). Middle East Journal of Agriculture Research 1, 33-39.

He JZ, Ru QM, Dong DD, Sun PL. 2012 - Chemical characteristics and antioxidant properties of crude water soluble polysaccharides from four common edible mushrooms. Molecules 17, 4373-4387.

Hu X, Liu C, Wang X, Jia D et al. 2017 - Hyperglycemic and anti-diabetic nephritis activities of polysaccharides separated from Auricularia auricular in diet-streptozotocin-induced diabetic rats. Experimental and Therapeutic Medicine 13, 352-358.

Hyde K, Bahkali A, Moslem M. 2010 - Fungi - an unusual source for cosmetics. Fungal Diversity 43, 1-9.

Hyde KD, Xu J, Rapior S, Jeewon R et al. 2019 - The amazing potential of fungi, 50 ways we can exploit fungi industrially. Fungal Diversity (In press).

Irawati D, Hayashi C, Takashima Y, Wedatama S et al. 2012 - Cultivation of the edible mushroom Auricularia polytricha using sawdustbased substrate made of three Indonesian commercial 
plantation species, Falcataria moluccana, Shorea sp., and Tectona grandis. Micología Aplicada International 24, 33-41.

Jeong H, Yang B-K, Jeong Y-T, Kim G-N et al. 2007 - Hypolipidemic effects of biopolymers extracted from culture broth, mycelia, and fruiting bodies of Auricularia auricula-judae in dietary-induced hyperlipidemic rats. Mycobiology 35, 16-20.

Jia D, Wang B, Li X, Peng W et al. 2017 - Proteomic analysis revealed the fruiting-body protein profile of Auricularia polytricha. Current Microbiology 74, 943-951.

Jo WS, Kim DG, Seok SJ, Jung HY et al. 2014 - The culture conditions for the mycelial growth of Auricularia auricula-judae. Journal of Mushrooms 12, 88-95.

Kadnikova IA, Costa R, Kalenik TK, Guruleva ON et al. 2015 - Chemical composition and nutritional value of the mushroom Auricularia auricula-judae. Journal of Food and Nutrition Research 3, 478-482.

Kakuta M, Sone Y, Umeda T, Misaki A. 1979 - Comparative structural studies on acidic heteropolysaccharides isolated from "Shirokikurage," fruit body of Tremella fuciformis Berk, and the growing culture of its yeast-like cells. Agricultural and Biological Chemistry 43, 1659-1668.

Kalač P. 2010 - Trace element contents in European species of wild growing edible mushrooms: a review for the period 2000-2009. Food Chemistry 122, 2-15.

Kalač P. 2013 - A review of chemical composition and nutritional value of wild-growing and cultivated mushrooms. Journal of the Science of Food and Agriculture 93, 209-218.

Kamalebo HM, Malale HNSW, Ndabaga CM, Degreef J et al. 2018 - Uses and importance of wild fungi: traditional knowledge from the Tshopo province in the Democratic Republic of the Congo. Journal of Ethnobiology and Ethnomedicine 14, 13.

Kavishree S, Hemavathy J, Lokesh B, Shashirekha M et al. 2008 - Fat and fatty acids of Indian edible mushrooms. Food Chemistry 106, 597-602.

Khan AA, Jahangir MM, Fozia GS, Ziaf K et al. 2016 - Nutritional and chemical profiles of Auricularia auricular mushrooms: A review. International Journal of Agricultural and Environmental Research 2, 185-191.

Khaskheli SG, Zheng W, Sheikh SA, Khaskheli AA et al. 2015 - Characterization of Auricularia auricula polysaccharides and its antioxidant properties in fresh and pickled product. International Journal of Biological Macromolecules 81, 387-395.

Kiho T, Ito M, Nagai K, Hara C et al. 1987 - Polysaccharides in fungi. XX. Structure and antitumor activity of a branched $(1 \rightarrow 3)-\beta$-D-glucan from alkaline extract of Yū ěr. Chemical and Pharmaceutical Bulletin 35, 4286-4293.

Kiho T, Miho S, Wang S, Katsuyuki N. 1991 - Polysaccharides in fungi. XXVI. Two branched $(1 \rightarrow 3)-\beta$-D-glucans from hot water extract of Yū ěr. Chemical and Pharmaceutical Bulletin 39, 798-800.

Kiho T, Sakai M, Ukai S, Hara C et al. 1985 - Anti-inflammatory effect of the polysaccharide from the fruit bodies of Auricularia species. Carbohydrate Research 142, 344-351.

Kim TI, Park SJ, Choi CH, Lee SK et al. 2004 - Effect of ear mushroom (Auricularia) on functional constipation. The Korean Journal of Gastroenterology (Taehan Sohwagi Hakhoe chi) 44, 34-41.

Klupp NL, Kiat H, Bensoussan A, Steiner GZ et al. 2016 - A double-blind, randomised, placebocontrolled trial of Ganoderma lucidum for the treatment of cardiovascular risk factors of metabolic syndrome. Scientific Reports 6, 29540.

Li L, Li J, Zou L, Bai SY et al. 2007 - RAPD analysis of genetic diversity of nine strains of Auricularia auricular cultivated in Heilongjiang Province. Journal of Forestry Research 18, 136-138.

Li L, Yang XY, Pan L, Su Y et al. 2019 - Comparing three methods of extraction of Auricularia auricula polysaccharides. Current Topics in Nutraceutical Research 17, 7-10. 
Li L, Zhong CH, Bian YB. 2014 - The molecular diversity analysis of Auricularia auricula-judae in China by nuclear ribosomal DNA intergenic spacer. Electronic Journal of Biotechnology 17, 27-33.

Li Z, Han D, Wang M, Zhang Y et al. 2018 - Optimization of the sample preparation procedure for the determination of trace elements in Auricularia auricula by inductively coupled plasmaoptical emission spectrometry. Analytical Letters 51, 1763-1773.

Li ZY, Yao XP, Liu B, Ha Nizaier R et al. 2015 - Auricularia auricular-judae polysaccharide attenuates lipopolysaccharide-induced acute lung injury by inhibiting oxidative stress and inflammation. Biomedical Reports 3, 478-482.

Lichtenstein AH, Appel LJ, Brands M, Carnethon M et al. 2006 - Diet and lifestyle recommendations revision 2006. Circulation 114, 82-96.

Linnakoski R, Reshamwala D, Veteli P, Cortina-Escribano M et al. 2018 - Antiviral agents from Fungi: Diversity, mechanisms and potential applications. Frontiers in Microbiology 9, 23252325.

Liu F, He Y, Sun G. 2009 - Determination of protein content of Auricularia auricula using near infrared spectroscopy combined with linear and nonlinear calibrations. Journal of Agricultural and Food Chemistry 57, 4520-4527.

Lu A, Yu M, Shen M, Fang Z et al. 2018a - Antioxidant and anti-diabetic effects of Auricularia auricular polysaccharides and their degradation by artificial gastrointestinal digestion Bioactivity of Auricularia auricular polysaccharides and their hydrolysates. Acta Scientiarum Polonorum Technologia Alimentaria 17, 277-288.

Lu A, Yu M, Shen M, Xu S et al. 2018b - Preparation of the Auricularia auricular polysaccharides simulated hydrolysates and their hypoglycaemic effect. International Journal of Biological Macromolecules 106, 1139-1145.

Ma Y, Wang C, Zhang Q, Peng X et al. 2018 - The effects of polysaccharides from Auricularia auricula (Huaier) in adjuvant anti-gastrointestinal cancer therapy: A systematic review and network meta-analysis. Pharmacological Research 132, 80-89.

Ma Z, Wang J, Zhang L. 2008 - Structure and chain conformation of $\beta$-glucan isolated from Auricularia auricula-judae. Biopolymers 89, 614-622.

Ma Z, Wang J, Zhang L, Zhang Y et al. 2010 - Evaluation of water soluble $\beta$-glucan from Auricularia auricular-judae as potential anti-tumor agent. Carbohydrate Polymers 80, 977983.

Maehara Y, Tsujitani S, Saeki H, Oki E et al. 2012 - Biological mechanism and clinical effect of protein-bound polysaccharide K (KRESTIN $\left.{ }^{\circledR}\right)$ : review of development and future perspectives. Surgery Today 42, 8-28.

Mau JL, Wu KT, Wu YH, Lin YP. 1998 - Nonvolatile taste components of ear mushrooms. Journal of Agricultural and Food Chemistry 46, 4583-4586.

Meng X, Liang H, Luo L. 2016 - Antitumor polysaccharides from mushrooms: a review on the structural characteristics, antitumor mechanisms and immunomodulating activities. Carbohydrate Research 424, 30-41.

Mironczuk-Chodakowska I, Witkowska AM, Zujko ME, Terlikowska KM. 2017 - Quantitative evaluation of 1,3,1,6 $\beta$-D-glucan contents in wild-growing species of edible Polish mushrooms. Roczniki Państwowego Zakładu Higieny 68, 281-288.

Misaki A, Kakuta M. 1995 - Kikurage (Tree-ear) and Shirokikurage (white Jelly-leaf): Auricularia auricula and Tremella fuciformis. Food Reviews International 11, 211-218.

Misaki A, Kakuta M, Sasaki T, Tanaka M et al. 1981 - Studies on interrelation of structure and antitumor effects of polysaccharides: antitumor action of periodate-modified, branched $(1 \rightarrow 3)-\beta$-D-glucan of Auricularia auricula-judae, and other polysaccharides containing $(1 \rightarrow 3)$-glycosidic linkages. Carbohydrate Research 92, 115-129.

Murata A, Oka N, Ohkubo S, Sato Y et al. 2004 - Adenosine as an active constituent of Auricularia auricula for the inhibition of rabbit platelet aggregation. Pharmacometrics 67, 359-364. 
Muszynska B, Grzywacz-Kisielewska A, Kala K, Gdula-Argasinska J. 2018 - Anti-inflammatory properties of edible mushrooms: A review. Food Chemistry 243, 373-381.

Nguyen TL, Chen J, Hu Y, Wang D et al. 2012a - In vitro antiviral activity of sulfated Auricularia auricula polysaccharides. Carbohydrate Polymers 90, 1254-1258.

Nguyen TL, Wang D, Hu Y, Fan Y et al. 2012b - Immuno-enhancing activity of sulfated Auricularia auricula polysaccharides. Carbohydrate Polymers 89, 1117-1122.

Ohiri RC, Bassey EE. 2017 - Evaluation and characterization of nutritive properties of the Jelly Ear culinary-medicinal mushroom Auricularia auricula-judae (Agaricomycetes) from Nigeria. International Journal of Medicinal Mushrooms 19, 173-177.

Onyango B, Palapala V, Arama P, Wagai S et al. 2011 - Nutritional analysis of some composted and non-composted agricultural substrates used for production of Kenyan native Wood Ear Mushrooms [Auricularia auricular (L. Ex Hook.) Underw.]. American Journal of Food Technology 10, 804-816.

Ooi VEC, Liu F. 2000 - Immunomodulation and anti-cancer activity of polysaccharide-protein complexes. Current Medicinal Chemistry 7, 715-729.

Pala SA, Wani AH, Bhat MY. 2013 - Ethnomycological studies of some wild medicinal and edible mushrooms in the Kashmir Himalayas (India). International Journal of Medicinal Mushrooms 15, 211-220.

Peng JT. 2008 - Agro-waste for cultivation of edible mushrooms in Taiwan. Food \& Fertilizer Technology Center Extension Bulletin 619. Paper presented at the International Workshop on Sustainable Utilization of Biomass and Other Organic Wastes as Renewable Energy Sources and for Agricultural and Industrial Uses, Tagaytay City, Philippines.

Priya RU, Geetha D, Darshan S. 2016 - Biology and cultivation of black ear mushroom Auricularia spp. Advances in Life Sciences 5, 10252-10254.

Qiu J, Zhang H, Wang Z, Liu D et al. 2018 - The antitumor effect of folic acid conjugatedAuricularia auricular polysaccharide-cisplatin complex on cervical carcinoma cells in nude mice. International Journal of Biological Macromolecules 107, 2180-2189.

Razak DLA, Abdullah N, Johari NMK, Sabaratnam V. 2013 - Comparative study of mycelia growth and sporophore yield of Auricularia polytricha (Mont.) Sacc. on selected palm oil wastes as fruiting substrate. Applied Microbiology and Biotechnology 97, 3207-3213.

Razak DLBA. 2013 - Cultivation of Auricularia polytricha Mont. Sacc. (Black jelly mushroom) using oil palm wastes. University of Malaya, Kuala Lumpur.

Reyes RG, Lopez L, Kumakura K, Kalaw SP et al. 2009 - Coprinus comatus, a newly domesticated wild nutriceutical mushroom in the Philippines. Journal of Agricultural Technology 5, 299-316.

Reza A, Choi MJ, Damte D, Jo WS et al. 2011 - Comparative antitumor activity of different solvent fractions from an Auricularia auricula-judae ethanol extract in P388D1 and sarcoma 180 cells. Toxicological Research 27, 77-83.

Reza MA, Hossain MA, Damte D, Jo WS et al. 2015 - Hypolipidemic and hepatic steatosis preventing activities of the wood ear medicinal mushroom Auricularia auricula-judae (higher Basidiomycetes) ethanol extract in vivo and in vitro. International Journal of Medicinal Mushrooms 17, 723-734.

Reza MA, Hossain MA, Lee SJ, Yohannes SB et al. 2014 - Dichloromethane extract of the jelly ear mushroom Auricularia auricula-judae (higher Basidiomycetes) inhibits tumor cell growth in vitro. International Journal of Medicinal Mushrooms 16, 37-47.

Reza MA, Jo WS, Park SC. 2012 - Comparative antitumor activity of jelly ear culinary-medicinal mushroom, Auricularia auricula-judae (Bull.) J. Schrot. (higher Basidiomycetes) extracts against tumor cells in vitro. International Journal of Medicinal Mushrooms 14, 403-409.

Royse DJ, Baars J, Tan Q. 2017 - Current overview of mushroom production in the world. In: Zied D C, Giménez A P (eds) Edible and medicinal mushrooms: Technology and applications. John Wiley \& Sons Ltd, West Sussex, UK, pp 5-13. 
Sánchez C. 2017 - Bioactives from mushroom and their application. In: Puri M (ed) Food bioactives. Springer, pp 23-57.

Sekara A, Kalisz A, Grabowska A, Siwulski M. 2015 - Auricularia spp. - mushrooms as novel food and therapeutic agents - a review. Sydowia 67, 1-10.

Sheu F, Chien PJ, Chien AL, Chen YF et al. 2004 - Isolation and characterization of an immunomodulatory protein (APP) from the Jew's Ear mushroom Auricularia polytricha. Food Chemistry 87, 593-600.

Shin CK, Yee CF, Shya LJ, Atong M. 2007 - Nutritional properties of some edible wild mushrooms in Sabah. Journal of Applied Sciences 7, 2216-2221.

Singdevsachan SK, Auroshree P, Mishra J, Baliyarsingh B et al. 2016 - Mushroom polysaccharides as potential prebiotics with their antitumor and immunomodulating properties: A review. Bioactive Carbohydrates and Dietary Fibre 7, 1-14.

Sone Y, Kakuta M, Misaki A. 1978 - Isolation and characterization of polysaccharides of "Kikurage," fruit body of Auricularia auricula-judae. Agricultural and Biological Chemistry 42, 417-425.

Song G, Du Q. 2010 - Isolation of a polysaccharide with anticancer activity from Auricularia polytricha using high-speed countercurrent chromatography with an aqueous two-phase system. Journal of Chromatography A 1217, 5930-5934.

Song G, Du Q. 2012a - Structure characterization and antitumor activity of an $\alpha \beta$-glucan polysaccharide from Auricularia polytricha. Food Research International 45, 381-387.

Song G, Du Q. 2012b - Structure characterization of a $\alpha \beta$-glucan polysaccharide from Auricularia polytricha. Natural Product Research 26, 1963-1970.

Stone BA. 2009 - Chemistry of $\beta$-Glucans. In: Bacic A, Fincher G, Stone B A (eds) Chemistry, Biochemistry, and Biology of $(1 \rightarrow 3)$ Beta Glucans and Related Polysaccharides. $1^{\text {st }}$ edn. Academic Press, San Diego, USA, pp 5-46.

Sun S, Zhang X, Chen W, Zhang L et al. 2016 - Production of natural edible melanin by Auricularia auricula and its physicochemical properties. Food Chemistry 196, 486-492.

Sun YX, Liu JC, Kennedy JF. 2010 - Purification, composition analysis and antioxidant activity of different polysaccharide conjugates (APPs) from the fruiting bodies of Auricularia polytricha. Carbohydrate Polymers 82, 299-304.

Tapingkae T. 2005 - Mushroom growing in Lao PDR. In: Mushroom Growers' Handbook 2 Shiitake Cultivation. MushWorld, Republic of Korea, pp 244-259.

Teke NA, Kinge TR, Bechem E, Nji TM et al. 2018 - Ethnomycological study in the Kilum-Ijim mountain forest, Northwest Region, Cameroon. Journal of Ethnobiology and Ethnomedicine 14, 25.

Thawthong A, Karunarathna SC, Thongklang N, Chukeatirote E et al. 2014 - Discovering and domesticating wild tropical cultivatable mushrooms. Chiang Mai Journal of Science 41, 731764.

Thongbai B, Rapior S, Hyde KD, Wittstein K et al. 2015 - Hericium erinaceus, an amazing medicinal mushroom. Mycological Progress 14, 91.

Tiwari BK, Pandey KB, Abidi AB, Rizvi SI. 2013 - Markers of oxidative stress during diabetes mellitus. Journal of Biomarkers 2013, Article ID 378790.

Ukai S, Hara C, Kuruma I, Tanaka Y. 1983a - Polysaccharides in fungi. XIV. Anti-inflammatory effect of the polysaccharides from the fruit bodies of several fungi. Journal of PharmacobioDynamics 6, 983-990.

Ukai S, Kiho T, Hara C, Morita M et al. 1983b - Polysaccharides in fungi. XIII. Antitumor activity of various polysaccharides isolated from Dictyophora indusiata, Ganoderma japonicum, Cordyceps cicadae, Auricularia auricula-judae, and Auricularia species. Chemical and Pharmaceutical Bulletin 31, 741-744.

Ukai S, Morisaki S, Goto M, Kiho T et al. 1982 - Polysaccharides in fungi. VII. Acidic heteroglycans from the fruit bodies of Auricularia auricula-judae Quel. Chemical and Pharmaceutical Bulletin 30, 635-643. 
USDA. 2018a - United States Department of Agriculture (USDA), Agricultural Research Service Full Report (All Nutrients): 11228, Jew’s ear, (pepeao), raw. National Nutrient Database for Standard Reference Legacy, The National Agricultural Library, USDA. https://ndb.nal.usda.gov/. Accessed 11 April 2018

USDA. 2018b - United States Department of Agriculture (USDA), Agricultural Research Service Full Report (All Nutrients): 11988, Fungi, Cloud ears, dried. National Nutrient Database for Standard Reference Legacy, The National Agricultural Library, USDA. https://ndb.nal.usda.gov/. Accessed 11 April 2018

Verma RK, Verma P. 2017 - Diversity of macro-fungi in central India-IV: Auricularia auriculajudae, a neutraceutical jelly mushroom. Van Sangyan: Tropical Forest Research Institute 4, 23-31.

Wang XM, Zhang J, Wu LH, Zhao YL et al. 2014 - A mini-review of chemical composition and nutritional value of edible wild-grown mushroom from China. Food Chemistry 151, 279-285.

Wasser S. 2002 - Medicinal mushrooms as a source of antitumor and immunomodulating polysaccharides. Applied Microbiology and Biotechnology 60, 258-274.

Wasser SP. 2017 - Medicinal mushrooms in human clinical studies. Part I. Anticancer, oncoimmunological, and immunomodulatory activities: A review. International Journal of Medicinal Mushrooms 19, 279-317.

Wu CY, Liang CH, Wu KJ, Shih HD et al. 2017 - Effect of different proportions of agrowaste on cultivation yield and nutritional composition of the culinary-medicinal jelly mushroom Auricularia polytricha (higher basidiomycetes). International Journal of Medicinal Mushrooms 19, 377-385.

Wu F, Yuan Y, Malysheva VF, Du P et al. 2014 - Species clarification of the most important and cultivated Auricularia mushroom "Heimuer": evidence from morphological and molecular data. Phytotaxa 186, 241-253.

Wu Q, Tan Z, Liu H, Gao L et al. 2010 - Chemical characterization of Auricularia auricula polysaccharides and its pharmacological effect on heart antioxidant enzyme activities and left ventricular function in aged mice. International Journal of Biological Macromolecules 46, 284-288.

Wu T, Xu B. 2015 - Antidiabetic and antioxidant activities of eight medicinal mushroom species from China. International Journal of Medicinal Mushrooms 17, 129-140.

Wu Z, Zhang M, Yang H, Zhou H et al. 2018 - Production, physico-chemical characterization and antioxidant activity of natural melanin from submerged cultures of the mushroom Auricularia auricula. Food Bioscience 26, 49-56.

Xiong W, Zhang Q, Yin F, Yu S et al. 2016 - Auricularia auricular polysaccharide-low molecular weight chitosan polyelectrolyte complex nanoparticles: Preparation and characterization. Asian Journal of Pharmaceutical Sciences 11, 439-448.

Xu CP, Yun JW. 2003 - Optimization of submerged-culture conditions for mycelial growth and exo-biopolymer production by Auricularia polytricha (wood ears fungus) using the methods of uniform design and regression analysis. Biotechnology and Applied Biochemistry 38, 193199.

Xu S, Zhang Y, Jiang K. 2016 - Antioxidant activity in vitro and in vivo of the polysaccharides from different varieties of Auricularia auricula. Food \& Function 7, 3868-3879.

Yan PS, Luo XC, Zhou Q. 2004 - RAPD molecular differentiation of the cultivated strains of the jelly mushrooms, Auricularia auricula and A. polytricha. World Journal of Microbiology and Biotechnology 20, 795-799.

Yang BK, Ha JY, Jeong SC, Jeon YJ et al. 2002 - Hypolipidemic effect of an exo-biopolymer produced from submerged mycelial culture of Auricularia polytricha in rats. Biotechnology Letters 24, 1319-1325.

Yang L, Zhao T, Wei H, Zhang M et al. 2011 - Carboxymethylation of polysaccharides from Auricularia auricula and their antioxidant activities in vitro. International Journal of Biological Macromolecules 49, 1124-1130. 
Yao FJ, Lu LX, Wang P, Fang M et al. 2018 - Development of a molecular marker for fruiting body pattern in Auricularia auricula-judae. Mycobiology 46, 72-78.

Yoon SJ, Yu MA, Pyun YR, Hwang JK et al. 2003 - The nontoxic mushroom Auricularia auricula contains a polysaccharide with anticoagulant activity mediated by antithrombin. Thrombosis Research 112, 151-158.

Yu J, Sun R, Zhao Z, Wang Y. 2014 - Auricularia polytricha polysaccharides induce cell cycle arrest and apoptosis in human lung cancer A549 cells. International Journal of Biological Macromolecules 68, 67-71.

Yu M, Xu X, Qing Y, Luo X et al. 2009 - Isolation of an anti-tumor polysaccharide from Auricularia polytricha (jew's ear) and its effects on macrophage activation. European Food Research and Technology 228, 477-485.

Yu YJ, Choi KH, Jeong JS, Lee GK et al. 2013 - Study on characteristic of mycelial culture in ear mushroom. Journal of Mushroom Science and Production 11, 15-20.

Yuan Z, He P, Cui J, Takeuchi H. 1998a - Hypoglycemic effect of water-soluble polysaccharide from Auricularia auricula-judae Quel. on genetically diabetic KK-Ay mice. Bioscience, Biotechnology, and Biochemistry 62, 1898-1903.

Yuan Z, He P, Takeuchi H. 1998b - Ameliorating effects of water-soluble polysaccharides from woody ear (Auricularia auricula-judae Quel.) in genetically diabetic KK-Ay mice. Journal of Nutritional Science and Vitaminology 44, 829-840.

Zent EL, Zent S, Iturriaga T. 2004 - Knowledge and use of fungi by a mycophilic society of the Venezuelan Amazon. Economic Botany 58, 214-226.

Zhang H, Wang ZY, Yang L, Yang X et al. 2011 - In vitro antioxidant activities of sulfated derivatives of polysaccharides extracted from Auricularia auricular. International Journal of Molecular Sciences 12, 3288-3302.

Zhang JX, Chen Q, Huang CY, Gao W et al. 2015a - History, current situation and trend of edible mushroom industry development. Mycosystema 34, 524-540.

Zhang M, Cui S, Cheung PCK, Wanga Q. 2007 - Antitumor polysaccharides from mushrooms: a review on their isolation process, structural characteristics and antitumor activity. Trends in Food Science \& Technology 18, 4-19.

Zhang M, Xiao G, Thring RW, Chen W et al. 2015b - Production and characterization of melanin by submerged culture of culinary and medicinal fungi Auricularia auricula. Applied Biochemistry and Biotechnology 176, 253-266.

Zhang Y, Zeng Y, Men Y, Zhang J et al. 2018a - Structural characterization and immunomodulatory activity of exopolysaccharides from submerged culture of Auricularia auricula-judae. International Journal of Biological Macromolecules 115, 978-984.

Zhang YX, Bau T, Ohga S. 2018b - Biological characteristics and cultivation of fruit body of wild edible mushroom Auricularia villosula. Kyushu University Institutional Repository 63, 5-14.

Zhao R, Yang W, Pei F, Zhao L et al. 2018 - In vitro fermentation of six kinds of edible mushrooms and its effects on fecal microbiota composition. LWT - Food Science and Technology, 627-635.

Zhao S, Rong C, Liu Y, Xu F et al. 2015 - Extraction of a soluble polysaccharide from Auricularia polytricha and evaluation of its anti-hypercholesterolemic effect in rats. Carbohydrate Polymers 122, 39-45.

Zou Y, Jiang A, Tian M. 2015 - Extraction optimization of antioxidant polysaccharides from Auricularia auricula fruiting bodies. Food Science and Technology 35, 428-433.

Zou Y, Ma K. 2018 - Screening of Auricularia auricula strains for strong production ability of melanin pigments. Food Science and Technology 38, 41-44. 